\title{
Identification of KIF23 as a prognostic signature for ovarian cancer based on large scale samples and clinical validation
}

\section{Yuexin Hu}

Department of Gynecology and Obstetrics, Shengjing Hospital of China Medical University, No. 36 Sanhao Street, Heping District, Shenyang 110004, Key Laboratory of Maternal-Fetal Medicine of Liaoning Province, Key Laboratory of Obstetrics and Gynecology of

\section{Mingjun Zheng}

Department of Gynecology and Obstetrics, Shengjing Hospital of China Medical University, No. 36 Sanhao Street, Heping District, Shenyang 110004, Key Laboratory of Maternal-Fetal Medicine of Liaoning Province, Key Laboratory of Obstetrics and Gynecology of

\section{Caixia Wang}

Department of Gynecology and Obstetrics, Shengjing Hospital of China Medical University, No. 36 Sanhao Street, Heping District, Shenyang 110004, Key Laboratory of Maternal-Fetal Medicine of Liaoning Province, Key Laboratory of Obstetrics and Gynecology of

\section{Shuang Wang}

Department of Gynecology and Obstetrics, Shengjing Hospital of China Medical University, No. 36 Sanhao Street, Heping District, Shenyang 110004, Key Laboratory of Maternal-Fetal Medicine of Liaoning Province, Key Laboratory of Obstetrics and Gynecology of

\section{Rui Gou}

Department of Gynecology and Obstetrics, Shengjing Hospital of China Medical University, No. 36 Sanhao Street, Heping District, Shenyang 110004, Key Laboratory of Maternal-Fetal Medicine of Liaoning Province, Key Laboratory of Obstetrics and Gynecology of

\section{Ouxuan Liu}

Department of Gynecology and Obstetrics, Shengjing Hospital of China Medical University, No. 36 Sanhao Street, Heping District, Shenyang 110004, Key Laboratory of Maternal-Fetal Medicine of Liaoning Province, Key Laboratory of Obstetrics and Gynecology of

\section{Xiao Li}

Department of Gynecology and Obstetrics, Shengjing Hospital of China Medical University, No. 36

Sanhao Street, Heping District, Shenyang 110004, Key Laboratory of Maternal-Fetal Medicine of Liaoning Province, Key Laboratory of Obstetrics and Gynecology of

\section{Juanjuan Liu}

Department of Gynecology and Obstetrics, Shengjing Hospital of China Medical University, No. 36 Sanhao Street, Heping District, Shenyang 110004, Key Laboratory of Maternal-Fetal Medicine of Liaoning Province, Key Laboratory of Obstetrics and Gynecology of 
Bei Lin ( $\sim$ linbei88@hotmail.com )

Shengjing Hospital of China Medical University https://orcid.org/0000-0002-8881-9859

\section{Research}

Keywords: KIF23, Ovarian cancer, Prognosis, Bioinformatics, Biomarker, Immunohistochemistry

Posted Date: February 11th, 2020

DOl: https://doi.org/10.21203/rs.2.23036/v1

License: (c) (i) This work is licensed under a Creative Commons Attribution 4.0 International License. Read Full License 


\section{Abstract}

Background: Ovarian cancer is one of the common malignant tumors in gynecology. Although the treatment strategy for ovarian cancer has been greatly improved in recent years, due to the metastasis, recurrence and drug resistance, the 5 -year overall survival rate of patients is still less than $47 \%$. However, at present, there is no specific markers for clinical application. The purpose of this study is to verify the expression and clinical significance of KIF23 in ovarian cancer and identify potential targets for the clinical treatment of ovarian cancer.

Methods: The expression of KIF23 in ovarian cancer tissues and its relationship between survival prognosis and clinical pathological parameters were analyzed in Oncomine, GEO, and TCGA databases. KIF23 expression was analyzed by Kaplan-Meier plotter database and its relationship with chemoresistance was studied. The molecular mechanism involved in KIF23 was analyzed from the perspective of gene mutation, copy number variation and other genomics. Finally, immunohistochemistry experiment was used to verify the expression of KIF2, and its relationship between the clinical pathological parameters and prognosis of ovarian cancer patients was analyzed by single factor and multivariate Cox regression models.

Results: Bioinformatic and experimental results have demonstrated that KIF23 is highly expressed in ovarian cancer, and its high expression is positively correlated with poor prognosis. Overexpression of KIF23 can cause chemotherapy resistance in ovarian cancer and affect the overall survival of patients. Genomics analysis showed that KIF23 expression was associated with mutations such as FLG2 and TTN, and it was significantly enriched in tumor signaling pathways such as DNA replication and cell cycle.

Conclusions: KIF23 can not only be used as a biomarker of poor prognosis in patients with various stages of ovarian cancer, but also be used as a molecular targeted drug and an independent prognostic biomarker for the treatment of ovarian cancer patients.

\section{Background}

Ovarian cancer has the highest fatality rate among gynecological malignant tumors [1]. Due to its unclear pathogenesis and lack of sensitive screening methods, 70 to $80 \%$ of patients are diagnosed with advanced-stage disease and have a 5 -year survival rate of less than $45 \%$. Therefore, exploring the mechanisms underlying the development and progression of ovarian cancer and identifying useful biomarkers for this disease are critical needs [2].

The kinesin protein superfamily (kinesin family, KIF) belongs to the class of molecular motors. Its globular head has ATPase activity, which can obtain energy by hydrolyzing ATP and changing its configuration [3]. Kinesins are involved in the transport of vesicles, organelles, chromosomes, and RNAbinding proteins in cells, the formation of spindles and intermediates, and the separation of chromosomes. Abnormal expression of KIF family members plays an important role in tumor development [4-5]. Kinesin family member 23 (KIF23), which belongs to the kinesin 6 family, is localized 
to the mitotic spindle region. It plays an important role in mitotic cytoplasmic separation [6-7]. High KIF23 expression levels affect normal cytokinesis and centrosome formation, which in turn leads to the arrest of cell division or abnormal division, resulting in aneuploid cells that cause tumorigenesis. High KIF23 expression can also regulate AKT activity, levels of phosphorylation and proteasome degradation pathway, leading to tumor invasion [8-9].

KIF23 is highly expressed in a variety of tumors, such as breast cancer, gastric cancer, and lung cancer. KIF23 overexpression is significantly associated with tumor grade, invasion, and prognosis in breast cancer [5]. The high expression of KIF23 in glioma cells may be related to transcriptional activation. KIF23 knockdown can significantly inhibit glioma cell proliferation in vitro and in vivo [10]. Zhao $\mathrm{C}$ et al found that KIF23 expression is significantly elevated in glioma samples. MiR-424 acts as a tumor suppressor and inhibits cell migration and EMT by targeting KIF23 in gliomas [11]. Murakami et al. [12] found that KIF23 expression levels are significantly elevated in gastric cancer and associated with poor prognosis. Kato et al. [13] found that KIF23 expression is significantly increased in NSCLC tissues, especially in adenocarcinoma tissues, and patients with high expression typically have a poor prognosis.

Previously, we found that KIF23 was a poor prognostic indicator for endometrial cancer [14]. In this study, we analyzed KIF23 expression in multiple databases and studied the correlation between its expression and clinical stage, tumor grade, and patient prognosis in ovarian cancer. Immunohistochemistry experiment was conducted in order to verify the conclusion. Finally, we explored the molecular pathways and functions of KIF23 involved in the development of ovarian cancer. Our results provide clues for understanding the roles and mechanisms of KIF23 in the development of ovarian cancer.

\section{Methods}

\section{Oncomine analysis}

The Oncomine database is a database of gene chips with an integrated data mining platform. In this database, the conditions for filtering and mining data can be set to accommodate specific needs. The screening conditions for the current study were as follows: \ "Cancer Type: Ovary cancer"; \ "Gene: KIF23"; $\bigotimes$ "Analysis Type: Cancer vs Normal Analysis"; $\otimes$ threshold setting conditions of $P<0.01$, fold-change $>2$, and gene rank is top $10 \%$.

\section{GEO analysis}

Three ovarian cancer datasets were analyzed from the GEO database (GSE14407[15], GSE18520[16], and GSE54388[17]). All three datasets were based on the GPL570 platform ([HG-U133_Plus_2] Affymetrix Human Genome U133 + 2.0 Array). GSE14407 contained 12 samples each of serous papillary ovarian cancer and normal ovarian epithelium. GSE18520 contained 53 high-grade serous papillary carcinoma samples and ten paracancerous samples. GSE54388 contained 16 well or moderately differentiated ovarian cancer samples and six normal ovarian epithelial samples. The datasets were processed, calibrated, standardized, and log2-converted using the R package. The KIF23 expression was extracted 
from the three datasets, and differential expression box plots were drawn using "ggpubr" in the $\mathrm{R}$ package.

\section{Kaplan-Meier Plotter for survival analysis}

The Kaplan-Meier Plotter database (http://kmplot.com) [18] contains 10188 cancer samples, including 4142 breast cancer, 1648 ovarian cancer, 2437 lung cancer, and 1065 gastric cancer patient samples, that allows the assessment of the impact of 54675 genes on patient survival. Patients with ovarian cancer were divided into two groups based on KIF23 expression levels. Survival analysis was performed using

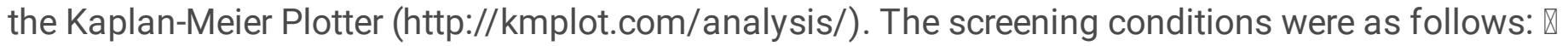
"Cancer: Ovary Cancer"; \ "Gene: KIF23"; \ "Survival: OS/PFS"; 『" Follow up threshold: 120 months". Subgroup conditions including clinical stage, tissue classification, and mutation status were defined in the database. The prognosis of ovarian cancer patients with different stages, tumor grades, and mutations was analyzed based on KIF23 expression levels. The hazard ratios (HRs) with $95 \%$ confidence intervals (Cls) and log rank p-values were generated.

\section{TCGA analysis}

An ovarian cancer dataset consisting of 374 tumor tissue samples was downloaded and pre-processed from the TCGA database (https://tcga-data.nci.nih.gov/tcga/). KIF23 expression was ranked from low to high, and the samples were divided into four equal parts. The first and last $25 \%$ of the samples were selected as the low expression and high expression groups, respectively.

\section{Function and Pathway Enrichment Analysis}

Proteins that interact with KIF23 were found in the cBioportal (http://www.cbioportal.org/) [19] database. Based on the p-values, the top 50 significantly related molecules were screened using DAVID Bioinformatics Resources (http://david.abcc. Ncifrrf.gov/) [20], Gene Ontology (GO) functional analysis was performed on the genes corresponding to the above proteins, and the significance of the enrichment to the Kyoto Encyclopedia of Genes and Genomes (KEGG) pathways was calculated using hypergeometric test according to the formula.

$$
\mathrm{P}=1-\mathrm{m}_{\mathrm{k}=0} \frac{\left(\begin{array}{l}
\mathrm{n} \\
\mathrm{k}
\end{array}\right)\left(\begin{array}{l}
\mathrm{N}-\mathrm{n} \\
\mathrm{M}-\mathrm{k}
\end{array}\right)}{\left(\begin{array}{l}
\mathrm{N} \\
\mathrm{M}
\end{array}\right)}
$$

In formula (1), $\mathrm{N}$ is the number of genome-wide genes, $\mathrm{M}$ is the number of genes annotated to a given pathway in the whole genome, $\mathrm{n}$ is the number of genes in the network, and $\mathrm{m}$ is the number of genes annotated to a given pathway.

\section{GSEA analysis}


GSEA analysis was performed using GSEA version 3.0 software. The c2.cp.kegg.v6.1.symbols.gmt dataset was downloaded from the MsigDB database on the GSEA website. The high-low grouping expression spectrum data and the attribute file were subjected to enrichment analysis by default weighted enrichment statistics, and the random combination number was set to 1000.

\section{Sample sources and clinical data}

Ovarian tissues $(n=167)$ were collected from surgical patients hospitalized at Shengjing Hospital of China Medical University from 2008 to 2015. All patients provided have signed informed consent forms, and complete clinical data were obtained for each patient. This study was approved by the Ethics Committee of China Medical University. None of the enrolled patients received radiotherapy, chemotherapy, or hormone therapy before surgery. All pathological sections were diagnosed by pathologists. The ovarian samples consisted of 115 epithelial ovarian cancer tumors (79 serous carcinomas, nine mucinous carcinomas, 18 endometrioid carcinomas, and nine clear cell carcinomas), 20 borderline epithelial ovarian tumors, 20 benign epithelial ovarian tumors, and 12 normal ovarian tissues. The average age of all patients was 53.52 years (16 to 84 years old). The median ages of patients with malignant, borderline, and benign tumors, and normal ovaries were 55 (16 to 79 years old), 52 (19 to 84 years old), 45 (28 to 79 years old), and 50.5 (35 to 67 years old), respectively. No statistically significant differences were noted between the groups. Of the 115 epithelial ovarian cancer samples, 62 were poorly differentiated, and 53 were moderately differentiated. Based on the International Federation of Obstetrics and Gynecology (FIGO, 2009), 47 cases were grades I-II, and 68 cases were grades III-IV. In addition, there were 28 cases of pelvic or para-aortic lymph node metastasis, while 87 had no metastasis.

\section{Immunohistochemistry}

Ovarian tissues were fixed in 10\% formalin and processed into 5 - $\propto \mathrm{m}$ thick paraffin sections. The samples were dewaxed with discontinuous concentrations of ethanol and blocked to inhibit endogenous peroxidase. The sections were heated in a microwave for antigen retrieval, cooled to room temperature, and blocked by incubation in goat serum for 30 minutes at $37^{\circ} \mathrm{C}$. Samples were incubated in rabbit antiKIF23 (Abcam, 1:200 dilution) overnight at $4^{\circ} \mathrm{C}$, followed by incubation with horseradish peroxidasecoupled goat anti-rabbit secondary antibody at $37^{\circ} \mathrm{C}$ for 30 minutes. Nuclei were stained blue by hematoxylin. Sections were then dehydrated, cleared by xylene, and mounted. The SP kit was used according to the manufacturer's instructions. Samples were deemed KIF23-positive when strong granular staining in the cell nucleus and cytoplasm was present.

Stained cells were classified based on their color intensity using the following scoring system: nonstaining, light yellow, brownish yellow, and dark brown, which were recorded as $0,1,2$, and 3 , respectively. Five fields were randomly evaluated for each slice using 400x magnification. The percentage of stained cells was also scored as follows: <5\% (0), 5 to 25\% (1), 26 to $50 \%$ (2), 51 to $75 \%$ (3), and > 75\% (4). The final score was calculated by multiplying the staining and percentage scores: 0 to $2(-), 3$ to 4 (+), 5 to 8 $(++)$, and 9 to 12 scores (+++). Final scores of 3 to 12 were considered positive expression, and 5 to 12 were considered high positive expression. 


\section{Statistical analysis}

Data were analyzed using SPSS 22.0 software (IBM Corporation, Armonk, NY, USA). Chi-squared and Fisher's exact tests were used to analyze counting data, and the t-test was used to analyze measurement data. Survival curves were analyzed by KM and log-rank tests. The Cox regression model was used to analyze the relationships between KIF23 expression and clinical data. $\mathrm{P}<0.05$ indicated statistically significant differences.

\section{Results}

\section{KIF23 expression in Oncomine and GEO databases}

A total of $447 \mathrm{KIF} 23$ studies in different types of cancer were collected from the Oncomine database (Fig. 1A). Of these studies, 67 studies showed statistically significant differences in the expression of KIF23 (64 studies showed significant increases; three studies showed significant decreases). Furthermore, analysis of KIF23 expression in independent ovarian cancer datasets [21-23], which contained 657 ovarian cancer samples and 22 normal samples, showed that KIF23 expression levels in all ovarian cancer groups were significantly higher than in the normal group $(P<0.01)$ (Fig. 1B-D). These results were verified using three independent ovarian cancer microarrays (GSE14407, GSE18520, and GSE54388) from the GEO database. Together, these results indicated that KIF23 expression in ovarian cancer tissues was significantly higher than that of adjacent non-cancerous tissues $(P<0.001)$ (Fig. 1E-G).

\section{Correlation between KIF23 mRNA expression and clinical pathological parameters in ovarian cancer using TCGA database}

To investigate the relationship between KIF23 expression and clinical-pathological parameters in ovarian cancer, we used the pathological data for ovarian cancer from the TCGA database, which contained the complete clinical data for 360 cases, including clinical stage, tumor grade, and age. Statistical analysis showed that KIF23 high expression was significantly correlated with poor differentiation $(P<0.05)$ (Table 1). However, KIF23 expression did not significantly correlate with the FIGO stage or age. Box plots of KIF23 expression and the clinical-pathological parameters are presented in Fig. 2A-C. 


\begin{tabular}{|lllll|}
\hline \multicolumn{5}{|c|}{ KIF23 expression } \\
\hline Item & Low & High & Total & $P$-value \\
\hline FIGO stage & & & & $P>0.05$ \\
\hline I II & 11 & 10 & 21 & \\
\hline III $\otimes$ IV & 202 & 137 & 339 & \\
\hline Grade & & & & \\
\hline Low Grade (1/2) & 37 & 9 & 46 & $P<0.05$ \\
\hline High Grade (3) & 186 & 128 & 314 & \\
\hline Age & & & & $P>0.05$ \\
\hline$>60$ & 117 & 54 & 271 & \\
\hline$<60$ & 115 & 74 & 189 & \\
\hline
\end{tabular}

Table 1. Relationship between KIF23 expression and clinical pathological parameters for malignant ovarian serous tumors in TCGA database

Table 2

Relationship between prognosis and KIF23 mRNA expression in patients with different stages of ovarian cancer

\begin{tabular}{|c|c|c|c|c|c|}
\hline \multicolumn{3}{|c|}{ PFS $(n=2000)$} & \multicolumn{3}{|c|}{ OS $(n=3241)$} \\
\hline Stage & Hazard Ratio & $\begin{array}{l}\mathrm{P}- \\
\text { value }\end{array}$ & Stage & Hazard Ratio & P-value \\
\hline $\begin{array}{l}I(n=107) \\
I+I(n=179) \\
I I(n=72) \\
I I I(n=1079) \\
I I I+I V(n= \\
1268)\end{array}$ & $\begin{array}{l}11.09(1.45- \\
84.95) \\
4.45(2.49-7.97) \\
2.78(1.39-5.57) \\
0.86(0.74-1.01) \\
0.85(0.74-0.98)\end{array}$ & $\begin{array}{l}0.004 \\
4.1 \mathrm{e}^{-} \\
08 \\
0.003 \\
0.060 \\
0.029\end{array}$ & $\begin{array}{l}I(n=107) \\
I+\|(n=179) \\
I I(n=72) \\
I I I(n=1079) \\
I I I+I V(n= \\
1268)\end{array}$ & $\begin{array}{l}21.65(2.74- \\
171.15) \\
6.15(1.83-20.7) \\
0.66(0.2-2.1) \\
1.38(1.17-1.62) \\
1.29(1.11-1.49)\end{array}$ & $\begin{array}{l}4.8 \mathrm{e}- \\
05 \\
0.001 \\
0.480 \\
0.000 \\
0.001\end{array}$ \\
\hline
\end{tabular}

\section{Relationship between KIF23 mRNA expression and ovarian cancer prognosis}

The censored data analyzed for overall survival (OS) consisted of 1,656 ovarian cancer cases. The overall survival of patients with high KIF23 expression was significantly worse than that of patients with low KIF23 expression (HR 1.31; $\mathrm{Cl} 1.13$ to 1.53; log-rank $\mathrm{P}=0.00032)$ (Fig. 3A). Analysis of 587 ovarian cancer cases (censored data) for the relationship between progression-free survival (PFS) and KIF23 expression 
yielded similar results (HR 1.27; $\mathrm{Cl} 1.12$ to 1.45 ; log-rank $\mathrm{P}=0.00026)$ (Fig. 3B). Thus, the disease-free survival time of patients with high KIF23 expression was significantly shorter than that of patients with low KIF23 expression. The analysis also demonstrated that patients with low KIF23 expression had a better prognosis for mutant TP53 ovarian cancer (Fig. 3C), while low KIF23 expression did not affect the prognosis of wild-type TP53 ovarian cancer patients (Fig. 3D).

\section{Relationship between KIF23 mRNA levels and prognosis of patients with different stages of ovarian cancer}

For PFS, higher KIF23 expression levels in early-stage ovarian cancer (stages I/II/I + II) were associated with a worse prognosis than low expression levels (Table 2, Fig. 4A-C). The HR for Stage I ovarian cancer was $11.09(\mathrm{Cl} 1.45$ to 84.95 ; log-rank $\mathrm{P}=0.004)$, indicating that KIF23 may be a negative prognostic indicator for lower stage ovarian cancer patients. The HR was less than 1 for stage III and IV ovarian cancer (Table 2, Fig. 4D-F). Similar results were obtained for OS. The HRs were 21.65 (Cl 2.74 to 171.15; log-rank $\mathrm{P}=0.000)$ for stage $\mathrm{I}, 6.15(\mathrm{Cl} 1.83$ to 20.7 ; log-rank $\mathrm{P}=0.001)$ for stage $\mathrm{I}+\mathrm{II}$, and $1.29(\mathrm{Cl} 1.11$ to 1.49; log-rank $P=0.001)$ for stage III + IV (Table 2, Fig. 4G, H). Based on these results, KIF23 could be used as an indicator of poor overall prognosis for all stages of ovarian cancer.

Table 3

Relationship between prognosis and KIF23 mRNA expression in patients with different grades of ovarian cancer

\begin{tabular}{|c|c|c|c|c|c|}
\hline \multicolumn{3}{|c|}{ PFS $(n=1822)$} & \multicolumn{3}{|c|}{ OS $(n=3386)$} \\
\hline Grade & Hazard Ratio & P-value & Grade & Hazard Ratio & P-value \\
\hline $\begin{array}{l}1(n=56) \\
1+2(n=381) \\
2(n=325) \\
3(n=1024)\end{array}$ & $\begin{array}{l}4.02(0.52-30.98) \\
1.43(1.05-1.94) \\
1.39(1.01-1.92) \\
0.85(0.72-1)\end{array}$ & $\begin{array}{l}0.150 \\
0.023 \\
0.043 \\
0.050\end{array}$ & $\begin{array}{l}1(n=56) \\
1+2(n=381) \\
2(n=325) \\
3(n=1024) \\
4(n=20)\end{array}$ & $\begin{array}{l}9.28(1.22-70.48) \\
1.54(1.08-2.19) \\
1.16(0.86-1.58) \\
1.17(0.99-1.38) \\
5.27(1.69-16.38)\end{array}$ & $\begin{array}{l}0.009 \\
0.016 \\
0.330 \\
0.065 \\
0.002\end{array}$ \\
\hline
\end{tabular}

\section{Relationship between KIF23 mRNA levels and prognosis of patients with different grades of ovarian cancer}

For PFS, higher KIF23 expression levels in grade $1+2$ were associated with a worse prognosis (Table 3 , Fig. 5A-C). The HR was 1.43 ( $\mathrm{Cl} 1.05$ to 1.94 ; log-rank $P=0.023$ ) for grade $1+2$ ovarian cancer and 0.85 (CI 0.72 to 1.00 ; log-rank $P=0.050$ ) for grade 3 (Fig. 5D). For OS, the HR for high KIF23 expression levels was $9.28(\mathrm{Cl} 1.22$ to 70.48 ; log-rank $\mathrm{P}=0.009)$ for grade 1 ovarian cancer and $1.54(\mathrm{Cl} 1.08$ to 2.19; logrank $\mathrm{P}=0.016)$ for grade $1+2$ (Table 3 , Fig. $5 \mathrm{E}, \mathrm{F}$ ). There were no significant differences between grades 2 and 3 (Table 3, Fig. 5G, H), and the HR was 5.27 (Cl 1.69 to 16.38; log-rank $P=0.002$ ) for grade 4. 
KIF23 expression in different ovarian tissues

\begin{tabular}{|c|c|c|c|c|c|c|c|}
\hline \multirow[t]{2}{*}{ Group } & \multirow[t]{2}{*}{$n$} & \multicolumn{2}{|c|}{ Low } & \multicolumn{2}{|c|}{ High } & \multirow{2}{*}{$\begin{array}{l}\text { Positive } \\
\text { rate ( } \%\end{array}$} & \multirow{2}{*}{$\begin{array}{l}\text { High } \\
\text { positive } \\
\text { rate ( \%) }\end{array}$} \\
\hline & & )$^{-}$ & )$^{+}$ & )$^{(++}$ & )$^{(+++}$ & & \\
\hline Malignant & 115 & 6 & 8 & 37 & 64 & $94.78^{*}$ & $87.83^{\#}$ \\
\hline Borderline & 20 & 12 & 6 & 2 & 0 & 40.00 & 10.00 \\
\hline Benign & 20 & 14 & 5 & 1 & 0 & 30.00 & 5.00 \\
\hline Normal & 12 & 9 & 3 & 0 & 0 & 25.00 & 0.00 \\
\hline
\end{tabular}

\section{Relationship between KIF23 mRNA expression and prognosis of platinum/paclitaxel-treated ovarian cancer patients}

For the analysis of OS, the total number of eligible ovarian cancer cases treated with platinum-based chemotherapy was 1409. High KIF23 expression resulted in an HR of 1.16 (Cl 1.01 to 1.35 ; log-rank $\mathrm{P}=$ 0.039) (Fig. 6A). The total number of ovarian cancer patients that received paclitaxel chemotherapy was 793. The HR for high KIF23 expression was 1.31 ( $\mathrm{Cl} 1.08$ to 1.58; log-rank $\mathrm{P}=0.0056$ ) (Fig. 6B). A total of 776 patients received platinum-paclitaxel combination chemotherapy. The HR for high KIF23 expression in these patients was 1.32 ( $\mathrm{Cl} 1.09$ to 1.61 ; log-rank $P=0.0042)$ (Fig. $6 \mathrm{C}$ ), suggesting that KIF23 overexpression could lead to chemotherapy resistance in ovarian cancer, which affects the overall survival of patients. KIF23 may represent a therapeutic target for the treatment of this disease.

For PFS, the analysis of the relationship of KIF23 expression and prognosis for 1259 ovarian cancer patients that received platinum-based chemotherapy yielded an HR of $1.19(\mathrm{Cl} 1.05$ to 1.36 ; $\log$-rank $\mathrm{P}=$ 0.0083) (Fig. 6D), suggesting that overexpression of KIF23 may cause resistance to platinum-based chemotherapy. However, KIF23 had no significant effect on the PFS of ovarian cancer patients treated with paclitaxel chemotherapy alone or platinum-paclitaxel combination therapy (Fig. 6E, F).

\section{KIF23 expression in different ovarian tissues using IHC}

To verify the results of the database analyses, we performed KIF23 IHC on 167 ovarian samples. KIF23 staining primarily occurred in both the nucleus and cytoplasm. The results showed that KIF23 expression was significantly upregulated in epithelial ovarian cancer (Fig. 7). The positive expression and high positive expression rates in the epithelial ovarian cancer group were $94.78 \%$ and $87.83 \%$, respectively, 
which was significantly higher than that of the borderline ( $40 \%$ and $10 \%$, respectively), benign ( $30 \%$ and $5 \%$, respectively), and normal ( $25 \%$ and $0 \%$ ) groups ( $P<0.05$ for all comparisons) (Table 4$)$.

Table 5

Relationship between KIF23 expression and clinicopathological parameters in patients with ovarian cancer

\begin{tabular}{|c|c|c|c|c|c|c|c|}
\hline \multirow[t]{2}{*}{ Items } & \multirow[t]{2}{*}{$\mathrm{n}$} & \multicolumn{2}{|c|}{ Low } & \multicolumn{2}{|c|}{ High } & \multirow{2}{*}{$\begin{array}{l}\text { High } \\
\text { positive } \\
\text { rate (\%) }\end{array}$} & \multirow{2}{*}{$\begin{array}{l}\mathrm{P} \text { - } \\
\text { value }\end{array}$} \\
\hline & & $(-)$ & $(+)$ & $(++)$ & $(+++)$ & & \\
\hline FIGO stage & & & & & & & $\begin{array}{l}<.05 \\
0.05\end{array}$ \\
\hline HII & 47 & 4 & 7 & 17 & 19 & 76.60 & \\
\hline III-IV & 68 & 2 & 1 & 20 & 45 & 95.59 & \\
\hline Differentiation & & & & & & & $\begin{array}{l}<.05 \\
0.05\end{array}$ \\
\hline Well-Moderate & 54 & 5 & 5 & 18 & 25 & 79.63 & \\
\hline Poorly & 61 & 1 & 3 & 19 & 39 & 95.08 & \\
\hline $\begin{array}{l}\text { Lymph node } \\
\text { metastasis }\end{array}$ & & & & & & & $\begin{array}{l}< \\
0.05\end{array}$ \\
\hline Yes & 28 & 0 & 0 & 10 & 18 & 100.00 & \\
\hline No & 87 & 6 & 8 & 27 & 46 & 83.91 & \\
\hline $\begin{array}{l}\text { Pathological } \\
\text { subtype }\end{array}$ & & & & & & & $\overrightarrow{0.05}$ \\
\hline Serous & 79 & 2 & 4 & 27 & 46 & 92.41 & \\
\hline Mucinous & 9 & 0 & 1 & 3 & 5 & 88.89 & \\
\hline Endometrioid & 18 & 2 & 2 & 4 & 10 & 77.78 & \\
\hline $\begin{array}{l}\text { Clear cell } \\
\text { carcinoma }\end{array}$ & 9 & 2 & 1 & 3 & 3 & 66.67 & \\
\hline
\end{tabular}

\section{Relationship between KIF23 mRNA levels and prognosis of ovarian cancer patients using IHC}

Follow up of 115 ovarian cancer patients occurred through June 2019. Univariate analysis demonstrated that KIF23 expression levels (HR 0.098; $\mathrm{Cl} 0.013$ to 0.711 ; log-rank $\mathrm{P}=0.022)$, FIGO stage (HR 0.192; Cl 
0.092 to 0.401 ; log-rank $P=0.000)$, degree of differentiation (HR 1.885; $\mathrm{Cl} 1.020$ to 3.484 ; log-rank $\mathrm{P}=$ 0.043), and lymph node metastasis (HR 0.268; $\mathrm{Cl} 0.141$ to 0.510 ; log-rank $\mathrm{P}=0.000$ ) significantly correlated with overall survival (Table 6). The 5-year survival time of the KIF23 high expression group was significantly lower than that of the KIF23 low expression group (Fig. 8A). Patients with stage III-IV disease had a shorter survival time than those with stage I-II (Fig. 8B). Patients with poorly differentiated tumors had shorter survival times than those with well or moderately differentiated tumors (Fig. 8C). Patients with lymph node metastasis had shorter survival times than patients with no metastasis (Fig. 8D). Multivariate analysis demonstrated that high KIF23 expression (HR 0.129; $\mathrm{Cl} 0.018$ to 0.955 ; log-rank $\mathrm{P}=$ 0.045), advanced clinical stage (HR 0.239; $\mathrm{Cl} 0.105$ to 0.545 ; log-rank $\mathrm{P}=0.001$ ), and poor differentiation (HR 0.532; $\mathrm{Cl} 0.267$ to 1.0579; log-rank $\mathrm{P}=0.035)$ were independent risk factors for poor prognosis (Table 6).

\begin{tabular}{|c|c|c|c|c|c|c|}
\hline \multirow[t]{2}{*}{ Variables } & \multicolumn{3}{|c|}{ Univariate analysis } & \multicolumn{3}{|c|}{ Multivariate analysis } \\
\hline & $\mathrm{HR}$ & $\begin{array}{l}95 \% \mathrm{Cl} \text { of } \\
\mathrm{HR}\end{array}$ & $\begin{array}{l}\mathrm{P}- \\
\text { value }\end{array}$ & $\mathrm{HR}$ & $\begin{array}{l}95 \% \mathrm{Cl} \text { of } \\
\mathrm{HR}\end{array}$ & $\begin{array}{l}\text { P- } \\
\text { value }\end{array}$ \\
\hline Age (<60 vs $\geq 60$ years) & 0.595 & $0.333 \sim 1.063$ & 0.079 & 0.653 & $0.358 \sim 1.192$ & 0.165 \\
\hline $\begin{array}{l}\text { KIF23 expression (low vs } \\
\text { high) }\end{array}$ & 0.098 & $0.013 \sim 0.711$ & 0.022 & 0.129 & $0.018 \sim 0.955$ & 0.045 \\
\hline FIGO stage (I-II vs III-IV) & 0.192 & $0.092 \sim 0.401$ & 0.000 & 0.239 & $0.105 \sim 0.545$ & 0.001 \\
\hline $\begin{array}{l}\text { Lymph node metastasis (yes } \\
\text { vs no) }\end{array}$ & 0.268 & $0.141 \sim 0.510$ & 0.000 & 0.532 & $0.267 \sim 1.057$ & 0.072 \\
\hline $\begin{array}{l}\text { Differentiation (poor vs } \\
\text { well-moderate) }\end{array}$ & 1.885 & $1.020 \sim 3.484$ & 0.043 & 1.982 & $1.051 \sim 3.739$ & 0.035 \\
\hline $\begin{array}{l}\text { Pathological subtype } \\
\text { (serous vs nonserous) }\end{array}$ & 0.508 & $0.245 \sim 1.051$ & 0.068 & 1.036 & $0.489 \sim 2.194$ & 0.926 \\
\hline
\end{tabular}

Table6. Cox regression analysis of overall survival of ovarian epithelial serous tumors

\section{Molecular mechanism of KIF23 involved in the development of ovarian cancer}

To explore the molecular mechanisms behind the involvement of KIF23 in the development of ovarian cancer, somatic mutations were analyzed in the TCGA database. Gene expression data were analyzed for 433 samples, and a total of 284 samples had overlapping mutations. The median KIF23 expression level was calculated. Low expression samples had KIF23 levels below the median, and high expression samples had KIF23 levels above the median. 
FLG2 mutation was significantly enriched in patients with low KIF23 expression, whereas TP53 mutations were significantly enriched in both KIF23 populations. In the high KIF23 expression group, the TTN mutant lower expression group was significantly enriched. Mutations in USH2A, OBSCN, NF1, MUC17, and DST have significant differences under different expression conditions (Fig. 9A, B). However, the role of these mutations in ovarian cancer has not been thoroughly investigated. Using KEGG pathway analysis, we found that the high KIF23 expression mutation group was significantly enriched for dopaminergic synapse, beta-alanine metabolism, and glycosaminoglycan degradation (Fig. 9C). We hypothesize that KIF23 regulates these pathways to influence the occurrence and development of ovarian cancer mutations.

Ovarian cancer copy number variation data obtained from XENA (https://xenabrowser.net/datapages/) was divided into deep deletion, shallow deletion, diploid, gain, and amplification groups and compared to KIF23 expression levels. Our analysis demonstrated that KIF23 expression was significantly increased as the copy number was amplified (Fig. 9H).

GSEA gene enrichment analysis revealed that high KIF23 expression samples were significantly enriched for DNA replication and cell cycle gene sets (Fig. 9D, E). Of the identified genes, 160 genes were closely related to KIF23 expression (Pearson $|R|>0.5$ using the TCGA ovarian cancer gene expression profile data). GO functional enrichment analysis was performed to explore the biological behavior and molecular function of these genes. We found that gene expression associated with KIF23 expression was mainly involved in the regulation of cell cycle phase transition, cell cycle checkpoint, G2/M transition of the cell cycle, and DNA replication (Fig. 9F). The heat map based on the expression of the KIF23 interacting genes, GO function, and tissue differentiation is presented in Fig. 9G. The data showed that the genes associated with KIF23 expression were highly correlated with these functional sets, and, like KIF23, these genes were expressed more strongly in poor differentiated ovarian cancer samples compared to well or moderately differentiated ovarian tumors.

\section{Discussion}

The kinesin superfamily (KIF) is involved in a variety of normal cellular biological activities, such as cell mitosis and intracellular transport of vesicles and organelles [24]. Overexpression of certain kinesins, such as Eg5, can induce excessive spindle separation, causing uneven distribution of genetic material, thereby forming aneuploid progeny cells, which are involved in cancer invasion and metastasis [25-26]. Downregulation of certain kinesins, such as KIF20B, can cause mitotic arrest or cytokinesis defects, triggering apoptosis through p53 or other signaling pathways $[25,27]$. Studies have shown that KIF23, as a member of the kinesin superfamily 6 , is highly expressed in gastric cancer and is positively correlated with pTNM stage and poor prognosis. Knocking down KIF23 can inhibit the proliferation of gastric cancer cells [28]. Sun [29] et al. confirmed that TCF-4 regulates KIF23 expression at the transcriptional level, knocking down KIF23 by CHIP and dual luciferase gene reports, and glioma cells show dual and multinucleated cell bodies, thereby inhibiting tumor cell proliferation [30]. Liu [31] have shown that KIF23 can promote the proliferation, migration and invasion of human hepatocellular carcinoma cells by 
activating Akt signal transduction pathway. It is shown that KIF23 plays an important role in the occurrence and development of tumors. The fatality rate of ovarian cancer ranks first among gynecological malignant tumors, and $4 / 5$ of the patients are diagnosed with advanced ovarian cancer [32]. At present, the pathogenesis of ovarian cancer is not clear, and there are few studies on KIF23 in ovarian cancer. Therefore, it is of great significance to explore the relationship between KIF23 and ovarian cancer. Through the joint analysis of Oncomine, GEO and TCGA database, the results showed that KIF23 was highly expressed in ovarian cancer, and the high expression of KIF23 was significantly related to tissue differentiation. The overall survival time of patients with high expression of KIF23 was significantly worse than that of patients with low expression of KIF23. The immunohistochemistry results showed that the high expression of KIF23 was significantly correlated with FIGO stage and tissue differentiation. Whether the high expression of KIF23 is related to FIGO stage is not consistent with the conclusion of bioinformatics analysis. We speculate that it may be related to sample size and ethnic differences. The results of survival analysis showed that the overall survival time of patients with high expression of KIF23 was significantly worse than that of patients with low expression, which was consistent with the conclusion of bioinformatics analysis. Therefore, we speculate that KIF23 can be used as a biomarker to guide the early clinical diagnosis and efficacy detection of ovarian cancer.

It is reported that TP53 mutations exist in more than $50 \%$ of the advanced epithelial serous ovarian cancers, and the frequency of TP53 mutations can be as high as $80 \%$ when using purified tumor samples for sequence analysis [33-36]. TP53 mutation is involved in the occurrence and development of epithelial serous ovarian cancer [37]. Therefore, we analyze the survival curve of ovarian cancer patients according to the status of TP53 in order to determine which patients can benefit from TP53 gene targeting testing. The results suggest that patients with low expression of KIF23 gene have a better prognosis in TP53 mutant ovarian cancer.

Zhang [38] study found that the presence of TP53 mutations in ovarian cancer aggravates genomic instability and promotes the expression of MDR1, which in turn activates chemotherapy resistance in ovarian cancer. Murakami $\mathrm{H}$ [39] et al isolated 9 up-regulated genes including KIF23 from paclitaxelresistant cell lines by microarray analysis, indicating that KIF23 may be involved in the process of paclitaxel resistance in patients with gastric cancer peritoneal metastasis. Therefore, we speculate that the expression of KIF23 may be related to chemotherapy resistance in ovarian cancer. Our results show that overexpression of KIF23 can lead to platinum/paclitaxel or combined drug resistance in patients with ovarian cancer, affecting the overall survival. For PFS, overexpression of KIF23 only leads to platinum chemotherapy resistance, but has no significance in paclitaxel chemotherapy resistance or platinum combined paclitaxel chemotherapy resistance of ovarian cancer patients. Therefore, targeted therapy for KIF23 can be regarded as a promising ovarian cancer treatment strategy.

To further explore the molecular mechanism of KIF23 involved in the occurrence and development of ovarian cancer. The results showed that FLG2 mutations were significantly enriched in patients with low KIF23 expression. TP53 mutations were significantly enriched in both high and low expression groups of KIF23. In KIF23 high expression group, TTN mutation was significantly richer than that in low expression 
group and the mutations of USH2A, OBSCN and NF1, MUC17, DST were significantly different under different expression conditions. Kunstman JW [40] found that there was USH2A mutation in anaplastic thyroid carcinoma by whole exon sequencing. The mutant peptides presented by II MHC (major histocompatibility complex) in cancer are important targets for cancer immunotherapy. Some studies have shown that TNN and USH2A can be used as the most common mutant genes with predictive effect on new antigens [41]. OBSCN mutation is closely related to breast cancer and colorectal cancer [42-43]. However, the role of these mutations in ovarian cancer is rarely discussed in existing studies. Based on these findings, we further discussed the KEGG pathway of gene mutation, and we found that the high expression mutant group of KIF23 gene was significantly enriched in Dopaminergic synapse, beta-Alanine metabolism, Glycosaminoglycan degradation and other pathways. Beta-Alanine plays an anti-tumor role by inhibiting the migration of cervical cancer and kidney tumor cells [44]. Glycosaminoglycan is involved in multiple signal cascades required for angiogenesis, invasion and metastasis [45]. Therefore, we suggest that KIF23 can affect the occurrence and development of ovarian cancer by regulating these pathways.

Copy number variation ((CNV)) is closely related to genetic and phenotypic diversity of cancer [46]. By identifying the copy number variation of the whole genome of ovarian cancer, the regions with frequent increase and decrease of copy number have been identified. In addition, high-level amplification of CCNE1, RB1, MYC, ERBB2, PIK3CA, EVI1, AKT2, NOTCH3 and FGFR1 genes can be used as a predictive marker of ovarian cancer [47-49]. However, the copy number of KIF23 in ovarian cancer has not been studied. Therefore, we analyzed the KIF23 copy number, and the results showed that KIF23 gene expression increased significantly with the amplification of sample copy number. Our results showed that the high expression of KIF23 in ovarian cancer is partly caused by copy number amplification. GSEA gene enrichment analysis showed that KIF23 highly expressed genes were significantly enriched in DNA replication and cell cycle gene concentration. The expression of genes related to KIF23 expression is mainly involved in biological behaviors such as regulation of cell cycle phase transition, cell cycle checkpoint, cell cycle G2UniM phase transition, G2UniM transition of mitotic cell cycle, DNA replication and so on.

\section{Conclusions}

In conclusion, KIF23 is highly expressed in epithelial ovarian cancer, and its high expression indicates a poor prognosis. KIF23 can be used as an independent marker to predict the prognosis of patients with ovarian cancer. The large sample analysis of bioinformatics analysis can avoid the error caused by the small sample size of a single study, and the immunohistochemical experiment makes the above results more convincing. Our study first found that patients with low expression of KIF23 have a better prognosis in TP53 mutant ovarian cancer. KIF23 can be used as a molecular targeted drug for the treatment of ovarian cancer by affecting OS and PFS. Through multi-omic analysis, it is the first time to identify that the expression of KIF23 in ovarian cancer is related to FLG2, TTN and other mutations, and it is significantly enriched in tumor signal pathways such as DNA replication and cell cycle. Our research not only lays a direction for the clinical treatment and molecular mechanism research of ovarian cancer, but 
also brings hope for the cure of ovarian cancer. In the later stage, we will enrich our research through cell function experiments.

\section{Abbreviations}

KIF23

Kinesin family member 23

GEO

Gene Expression Omnibus

TCGA database

The Cancer Genome Atlas database

OS

overall survival

PFS

progression-free survival

HR

hazard ratio

$\mathrm{Cl}$

confidence interval

FIGO

International Federation of Obstetrics and Gynecology

\section{Declarations}

\section{Acknowledgement}

We sincerely thank BioMed Proofreading $\Theta^{\circledR}, L L C$ for guidance with the English copyediting of our paper.

\section{Authors' contributions}

HYX performed the majority of the work described here. ZMJ and HYX wrote and edited the manuscript. WCX and WS translated the manuscript. GR and LOX collected the data. HYX and LX performed the experiment. LJJ examined the data. LB supervised the project. All authors read and approved the final manuscript.

\section{Funding}

This work was supported by grants from National Natural Science Foundation of China (No. 81672590, No.81472437), Shengjing Freedom researchers' plan (201804).

\section{Availability of data and materials}

Not applicable. 


\section{Ethics approval and consent to participate}

Not applicable.

\section{Consent for publication}

All the authors have read and approved the paper and declare no potential conflicts of interest in the paper. If the paper is accepted, all the authors will observe the terms of the license to publish.

\section{Competing interests}

The authors declare that there are no conflicts of interest.

\section{References}

1. Torre LA, Bray F, Siegel RL, Ferlay J, Lortet-Tieulent J, Jemal A. Global cancer statistics, 2012[J]. CA Cancer J Clin.2015; 65(2):87-108.

2. Chou JL, Su HY, Chen LY, et al. Promoter hypermethylation of FBX032, a novel TGFbeta/SMAD4 target gene and tumor suppressor, is associated with poor prognosis in human ovarian cancer.Lab Invest. 2010; 90(3):414-425.

3. Hirokawa N, Noda Y, Tanaka Y, Niwa S. Kinesin superfamily motor proteins and intracellular transport. Nat Rev Mol. Cell. Biol. 2009; 10: 682-696.

4. Hirokawa N, Tanaka Y. Kinesin superfamily proteins (KIFs): Various functions and their relevance for important phenomena in life and diseases. Exp Cell Res. 2015; 334(1): 16-25. 6.

5. Zou JX, Duan Z, Wang J, et al. Kinesin family deregulation coordinated by bromodomain protein ANCCA and histone methyltransferase MLL for breast cancer cell growth, survival, and tamoxifen resistance. Mol Cancer Res.2014;12 (4): 539-549.

6. Rath O, Kozielski F. Kinesins and cancer. Nat Rev Cancer. 2012;12: 527-539.

7. Zhu C, Bossy-Wetzel E, Jiang W. Recruitment of MKLP1 to the spindle midzone/midbody by INCENP is essential for midbody formation and completion of cytokinesis in human cells. The Biochemical Journal. 2005; 389:373-381.

8. Liu X, Erikson RL. The nuclear localization signal of mitotic kinesin-like protein Mklp-1: effect on Mklp-1 function during cytokinesis. Biochem Biophys Res Commun. 2006; 353(4): 960-964.

9. Sablin EP. Kinesins and microtubules: Their structures and motor mechanisms[J]. Curr Opin Cell Biol.2000; 12(1): 35-41.

10. Takahashi S, Fusaki N, Ohta S, et al. Downregulation of kif23 suppresses glioma proliferation. Journal of Neuro-Oncology.2012;106(3):519-529.

11. Zhao C, Wang XB, Zhang YH, et, al.MicroRNA-424 inhibits cell migration, invasion and epithelialmesenchymal transition in human glioma by targeting KIF23 and functions as a novel prognostic predictor. Eur Rev Med Pharmacol Sci. 2018; 22(19): 6369-6378. 
12. Murakami $\mathrm{H}$, Ito $\mathrm{S}$, Tanaka $\mathrm{H}$, et al. Establishment of new intraperitoneal paclitaxel-resistant gastric cancer cell lines and comprehensive gene expression analysis. Anticancer Res. 2013; 33(10): 42994307.

13. Kato $\mathrm{T}$, Wada $\mathrm{H}$, Patel $\mathrm{P}$, et al. Overexpression of KIF23 predicts clinical outcome in primary lung cancer patients. Lung Cancer. 2016; 92: 53-61.

14. Zheng MJ, Gou R, Zhang WC, et al. Screening of prognostic biomarkers for endometrial carcinoma based on a ceRNA network. PeerJ. 2018; 6: e6091.

15. Lanczky A, Nagy A, Bottai G, et al. miRpower: a web-tool to validate survival-associated miRNAs utilizing expression data from 2,178 breast cancer patients. Breast. Cancer Res Treat. 2016;160(3): 439-446.

16. Bowen NJ, Walker LD, Matyunina LV, et al. Gene expression profiling supports the hypothesis that human ovarian surface epithelia are multipotent and capable of serving as ovarian cancer initiating cells. BMC Med Genomics.2009;12(29)2:71.

17. Mok SC, Bonome T, Vathipadiekal V, et al. A gene signature predictive for outcome in advanced ovarian cancer identifies a survival factor: microfibril-associated glycoprotein 2. Cancer Cell. 2009; 16(6): 521-32.

18. Yeung TL, Leung CS, Wong KK, et al. ELF3 is a negative regulator of epithelial-mesenchymal transition in ovarian cancer cells. Oncotarget.2017; 8(10):16951-16963.

19. Gao J, Aksoy BA, Dogrusoz U, Dresdner, GB. Integrative analysis of complex cancer genomics and clinical profiles using the cBioPortal. Sci Signal. 2013; 6: I1.

20. Huang DW, Sherman BT, Lempicki RA. Systematic and integrative analysis of large gene lists using DAVID Bioinformatics Resources. Nature Protoc. 2009; 4(1): 44-57.

21. Welsh JB, Zarrinkar PP, Sapinoso LM, et al. Analysis of gene expression profiles in normal and neoplastic ovarian tissue samples identifies candidate molecular markers of epithelial ovarian cancer. Proc Natl Acad Sci USA. 2001; 98(3): 1176-1181.

22. Yoshihara K, Tajima A, Dai K, et al. Gene expression profiling of advanced-stage serous ovarian cancers distinguishes novel subclasses and implicates zeb2 in tumor progression and prognosis. Cancer Science.2010; 100(8):1421-1428.

23. TCGA (The Cancer Genome Atlas): Ovarian Serous Cystadenocarcinoma Gene Expression Data.

24. Goldstein LS, Philp AV. The road less traveled: emerging principles of kinesin motor utilization. Annu Rev Cell Dev Biol. 1999; 15: 141- 83.

25. Castillo A, Morse HC, Godfrey VL, Naeem R, Justice MJ. Overexpression of Eg5 causes genomic instability and tumor formation in mice. Cancer Res. 2007; 67: 10138- 47.

26. Wordeman L. How kinesin motor proteins drive mitotic spindle function: lessons from molecular assays. Semin Cell Dev Biol. 2010; 21: 260-8.

27. Liu XR, Cai Y, Cao X et al. A new oncolytic adenoviral vector carrying dual tumour suppressor genes shows potent anti-tumour effect. J Cell Mol Med. 2012; 16: 1298- 309. 
28. Li XL, Ji YM, Song R, Li XN, Guo LS. KIF23 Promotes Gastric Cancer by Stimulating Cell Proliferation. Dis Markers. 2019; 2019.

29. Sun L, Zhang C, Yang Z, Wu Y, Wang H, Bao Z, and Jiang T. KIF23 is an independent prognostic biomarker in glioma, transcriptionally regulated by TCF-4. Oncotarget.2016; 7(17): 24646-55.

30. Takahashi S, Fusaki N, Ohta S, et al. Downregulation of KIF23 suppresses glioma proliferation. J Neurooncol.2012; 106(3): 519-29.

31. Liljeholm M, Irvine AF, Vikberg AL, et al. Congenital dyserythropoietic anemia type III (CDA III) is caused by a mutation in kinesin family member, KIF23. Blood. 2013; 6(6): 121(23).

32. Siegel RL, Miller KD, Jemal A. Cancer statistics, 2018. CA Cancer J Clin. 2018; 68(1):7-30.

33. Kupryjanczyk J, Thor AD, Beauchamp R et p53 gene mutations and protein accumulation in human ovarian cancer. Proc Natl Acad Sci USA.1993; 90: 4961-4965.

34. Wen WH, Reles A, Runnebaum IB, et al. p53 mutations and expression in ovarian cancers: correlation with overall survival. Int J Gynecol Pathol.1999; 18(1).

35. Chan WY, Cheung KK, Schorge JO, et al. Bcl-2 and p53 protein expression, apoptosis, and p53 mutation in human epithelial ovarian cancers. Am J Pathol. 2000; 156: 409-417.

36. Salani R, Kurman RJ, Giuntoli R, et Assessment of TP53 mutation using purified tissue samples of ovarian serous carcinomas reveals a higher mutation rate than previously reported and does not correlate with drug resistance. Int J Gynecol Cancer. 2008; 18(3).

37. Kindelberger DW, Lee Y, Miron A, et al. Intraepithelial carcinoma of the fimbria and pelvic serous carcinoma: Evidence for a causal relationship. Am J Surg Pathol. 2007; 31(2):161-169.

38. Zhang M, Zhuang G, Sun X, et al. TP53 mutation-mediated genomic instability induces the evolution of chemo-resistance and recurrence in epithelial ovarian cancer. Diagn Pathol. 2017; 12(1): 16.

39. Vikberg AL, Vooder T, Lokk K, Annilo K, Golovleva I. KIF23 mutation analysis and copy number alterations of KIF23 in non-small-cell lung cancer exhibiting over-expression. Onco Targets Ther. 2017; 10: 4969-4979.

40. Kunstman JW, Juhlin CC, Goh G, et al. Characterization of the mutational landscape of anaplastic thyroid cancer via whole-exome sequencing. Hum Mol Genet. 2015; 24(8): 2318-29.

41. Cai W, Zhou D, Wu W, et al. MHC class II restricted neoantigen peptides predicted by clonal mutation analysis in lung adenocarcinoma patients: implications on prognostic immunological biomarker and vaccine design. BMC Genomics.2018; 19(1): 582.

42. Sjöblom T, Jones $S$, Wood LD, et al. The consensus coding sequences of human breast and colorectal cancers. Science.2006; 314: 268-274.

43. Liu Y, Hu Z, DeLisi C. Mutated Pathways as a Guide to Adjuvant Therapy Treatments for Breast Cancer. Mol Cancer Ther. 2016; 15: 184-189.

44. Pandurangan $M$, Enkhtaivan G, Mistry B, et al. $\beta$-Alanine intercede metabolic recovery for amelioration of human cervical and renal tumors. Amino Acids. 2017; 49(8): 1373-1380. 
45. Afratis N, Gialeli C, Nikitovic D, et al. Glycosaminoglycans: Key players in cancer cell biology and treatment. FEBS J. 2012; 279(7).

46. Conrad DF, Pinto D, Redon R, et al. Origins and functional impact of copy number variation in the human genome. Nature. 2010; 464: 704-712.

47. Etemadmoghadam D, deFazio A, Beroukhim R, et al. Integrated genome-wide DNA copy number and expression analysis identifies distinct mechanisms of primary chemo-resistance in ovarian carcinomas. Clinical cancer research. 2009; 15:1417-1427.

48. Kim SW, Kim JW, Kim YT, et al. Analysis of chromosomal changes in serous ovarian carcinoma using high-resolution array comparative genomic hybridization: Potential predictive markers of chemoresistant disease. Genes, chromosomes \& cancer. 2007; 46: 1-9.

49. Gorringe KL, Jacobs S, Thompson ER, et al. High-resolution single nucleotide polymorphism array analysis of epithelial ovarian cancer reveals numerous microdeletions and amplifications. Clinical cancer research. 2007; 13: 4731-4739.

\section{Figures}

A

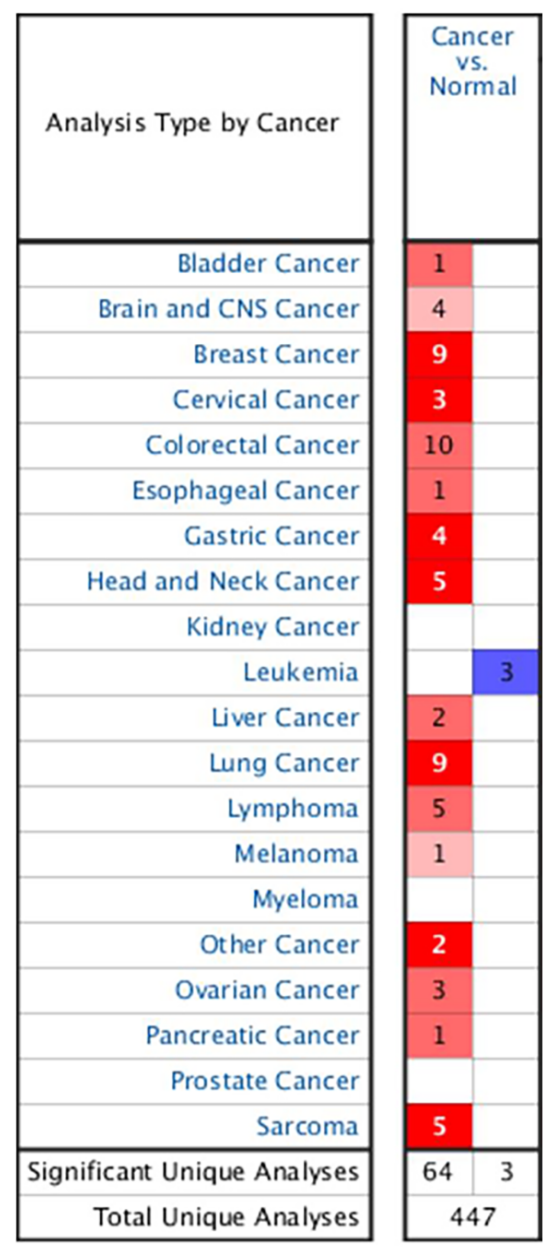

B

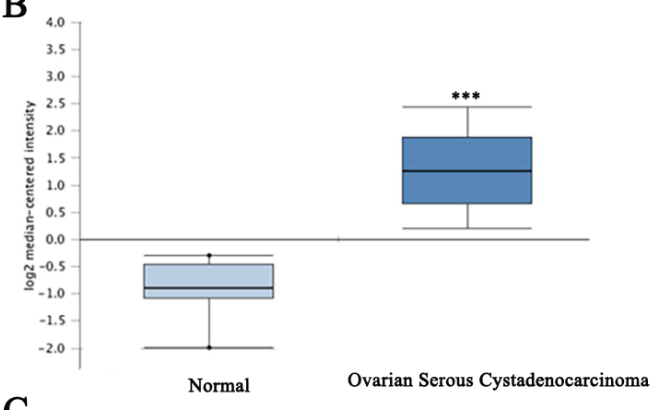

C

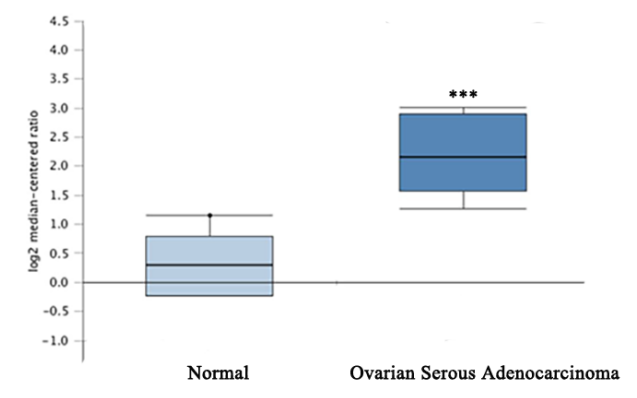

D

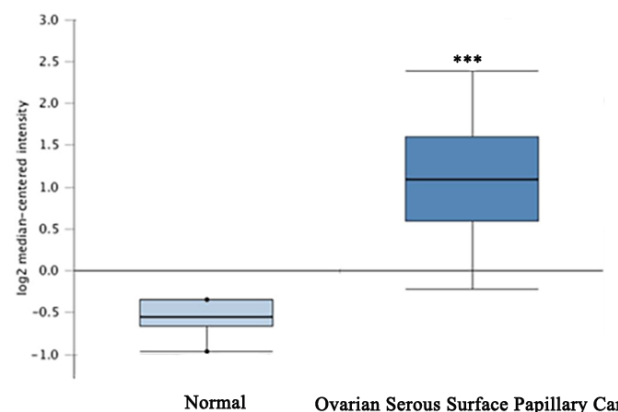

$\mathrm{E}$

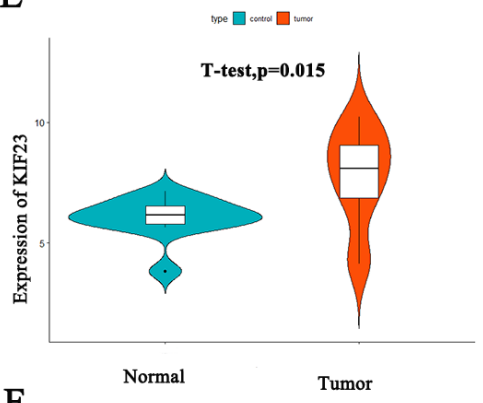

$\mathrm{F}$
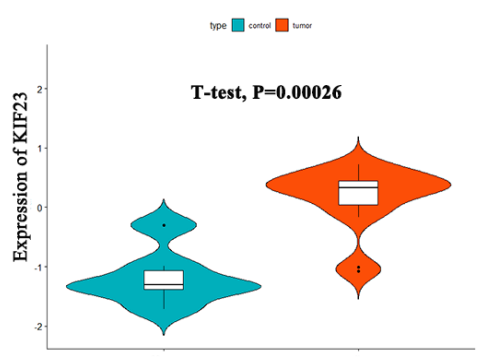

G

Normal

Tumor

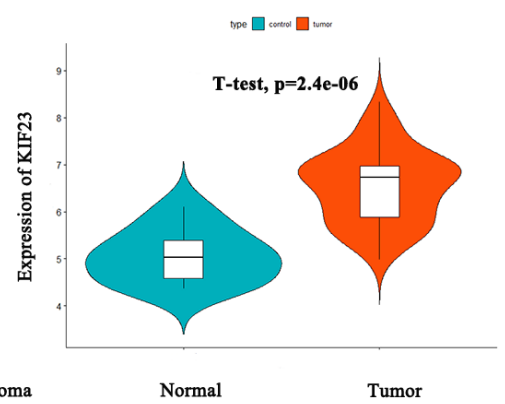


Figure 1

KIF23 expression in Oncomine and GEO databases. A. KIF23 expression in all tumor studies in the Oncomine database. B-D. Differential expression of KIF23 in the ovarian cancer dataset of the Oncomine database ( $* \star * P<0.01)$. E-G. KIF23 expression in GSE14407, GSE54388, and GSE18520 from the GEO database.

A

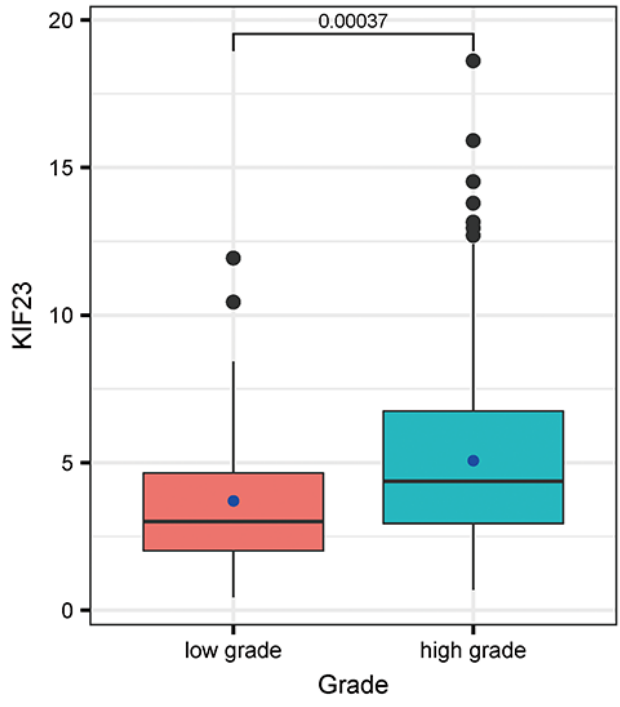

B

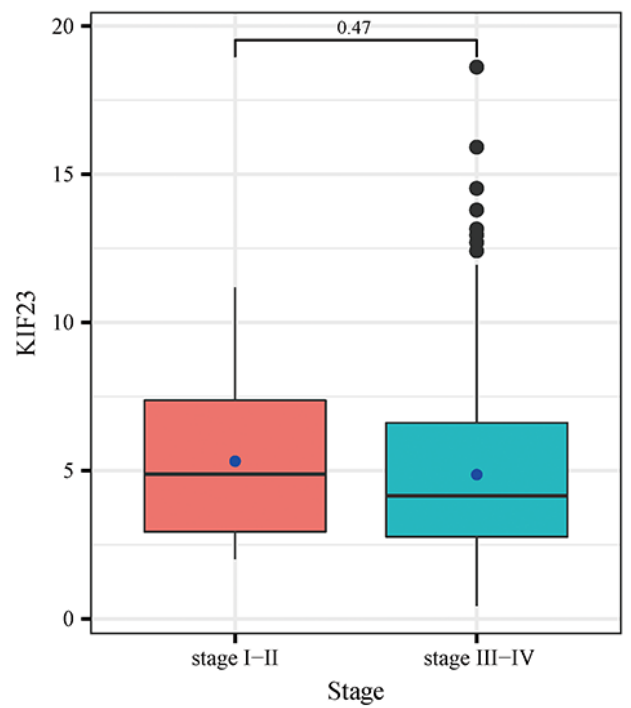

$\mathrm{C}$

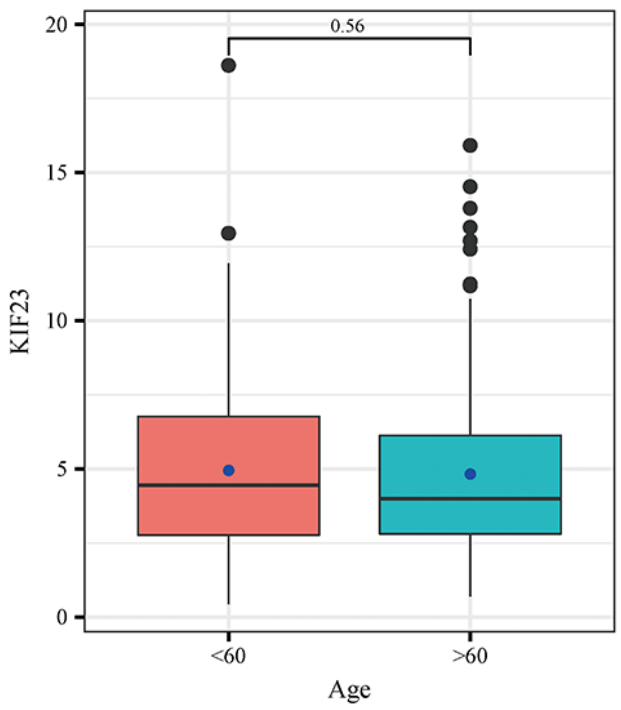

\section{Figure 2}

Box plots for the correlations between KIF23 expression and clinical pathological parameters. A. Relationship between KIF23 expression and grade B. Relationship between KIF23 expression and stage. C. Relationship between KIF23 expression and age. 
A

204709_s_at

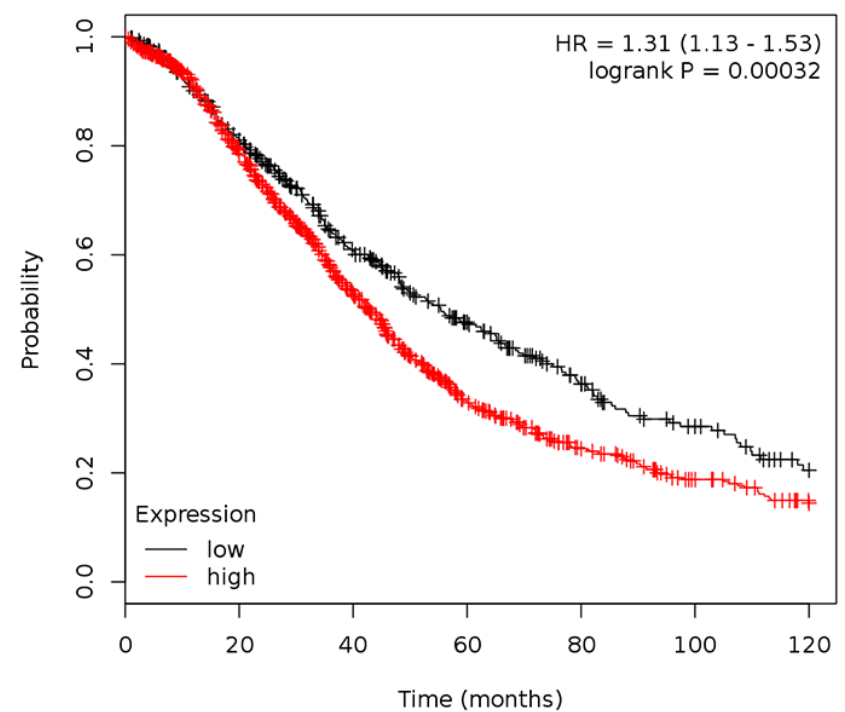

\begin{tabular}{ccccccc}
\multicolumn{8}{c}{ Number at risk } \\
low 432 & 318 & 193 & 116 & 68 & 41 & 21 \\
high 1224 & 832 & 429 & 194 & 104 & 56 & 31
\end{tabular}

$\mathrm{C}$

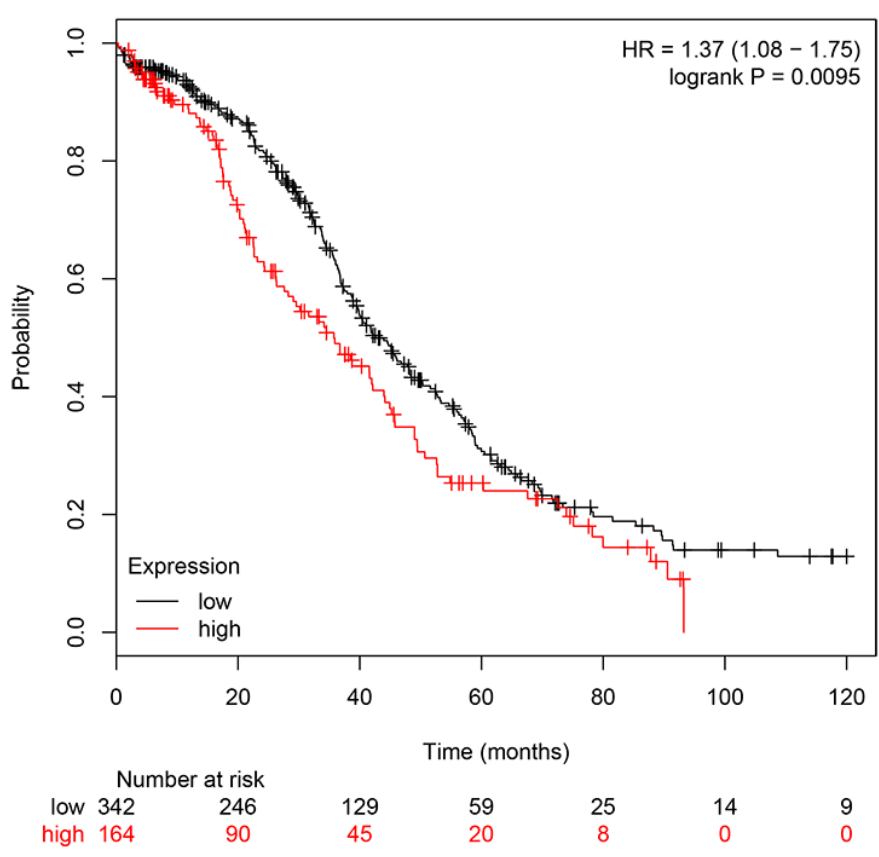

B

204709_s_at

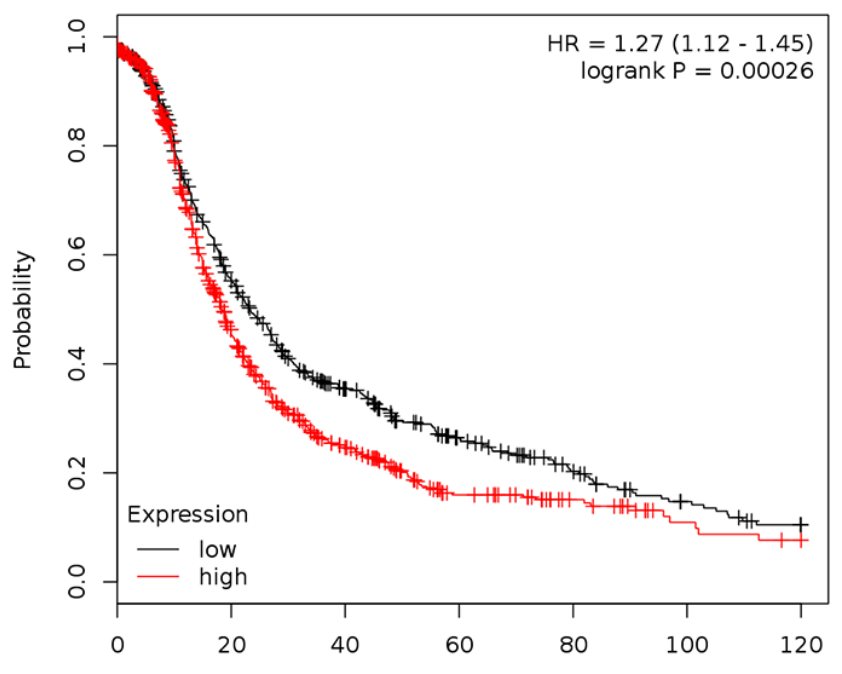

Time (months)

\begin{tabular}{ccccccc}
\multicolumn{9}{c}{ Number at risk } \\
low 580 & 283 & 143 & 77 & 46 & 25 & 14 \\
high 855 & 304 & 121 & 46 & 25 & 10 & 6
\end{tabular}

D

204709_s_at

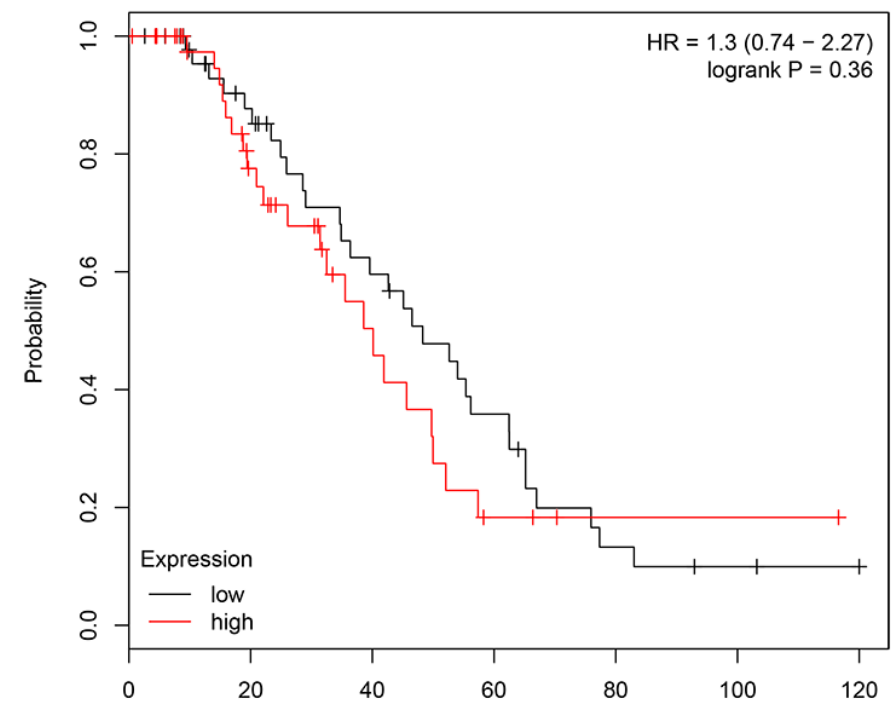

Time (months)

Number at risk

$\begin{array}{rrr}\text { low } & 48 & 34 \\ \text { high } & 46 & 25\end{array}$

\section{Figure 3}

Relationship between prognosis and KIF23 gene expression and TP53 mutations in ovarian cancer patients. 

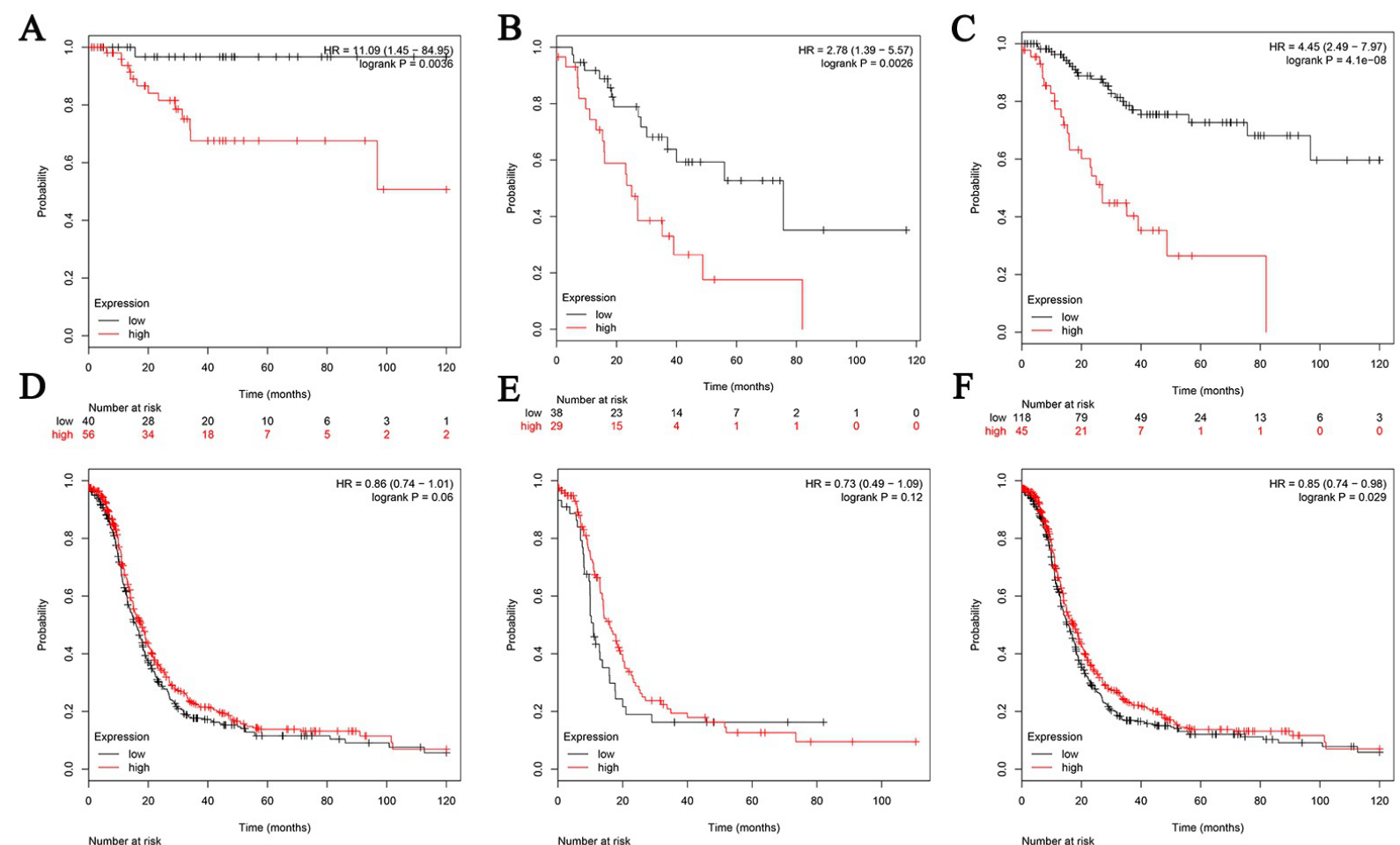

G

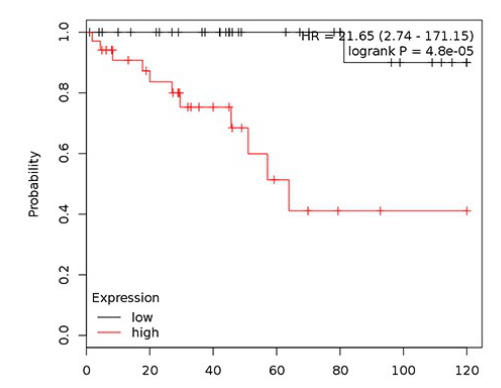

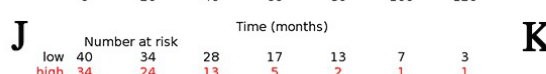

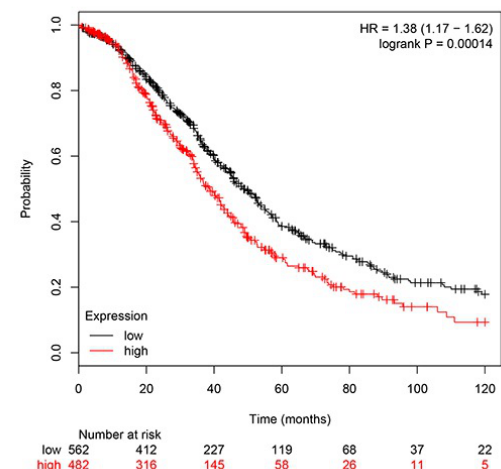

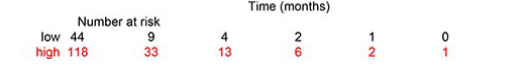

H
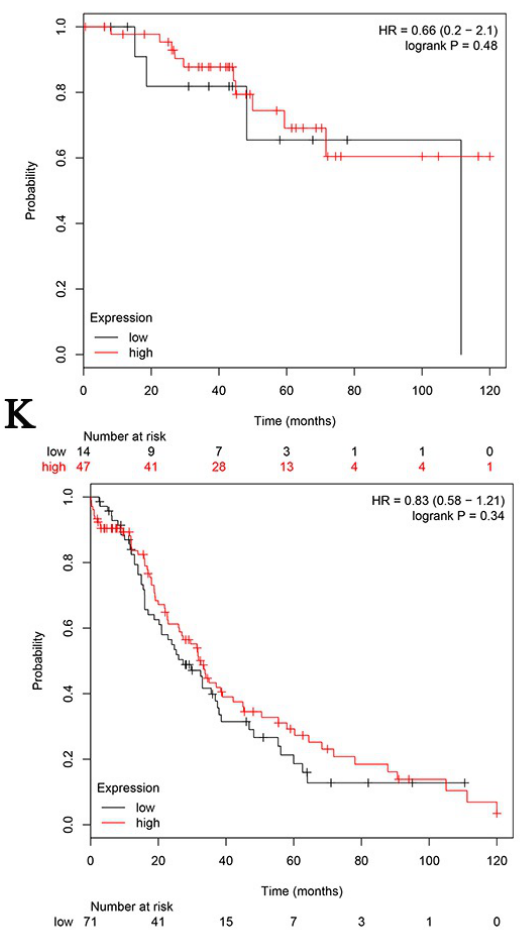

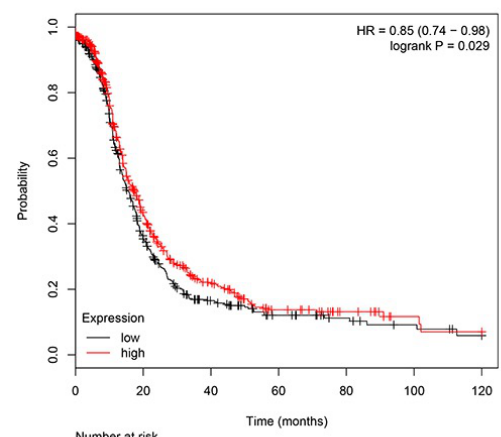

I
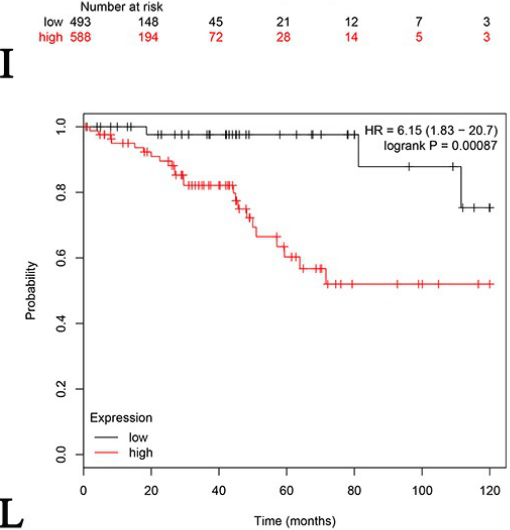

L

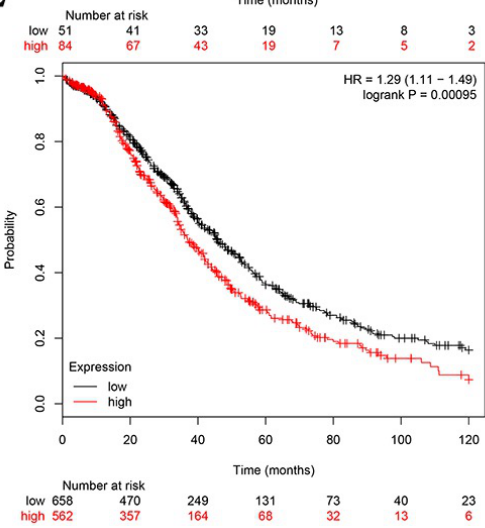

Figure 4

Relationship between prognosis and KIF23 mRNA expression in patients with different stages of ovarian cancer 

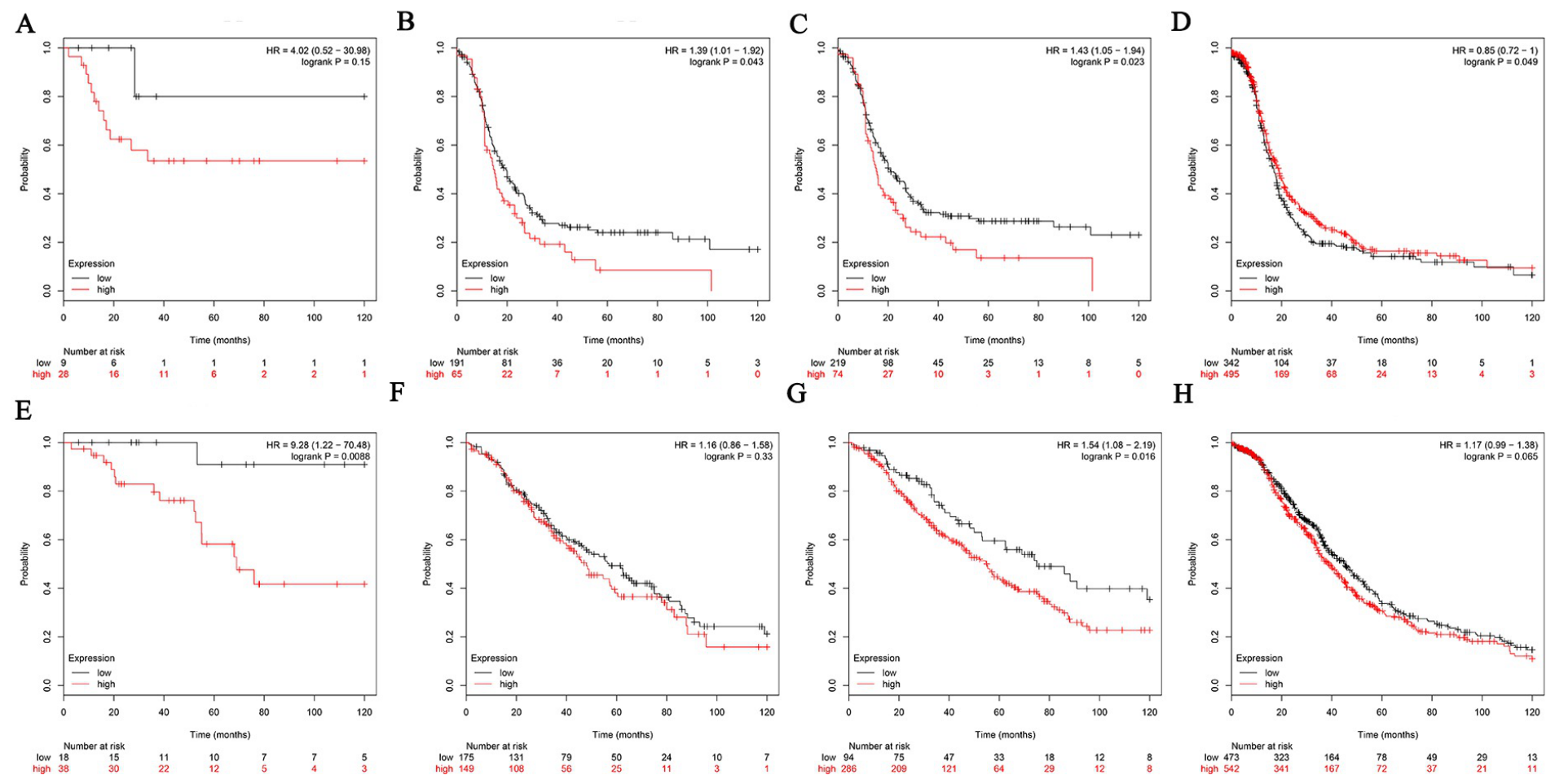

Figure 5

Relationship between prognosis and KIF23 mRNA levels in patients with different grades of ovarian cancer 

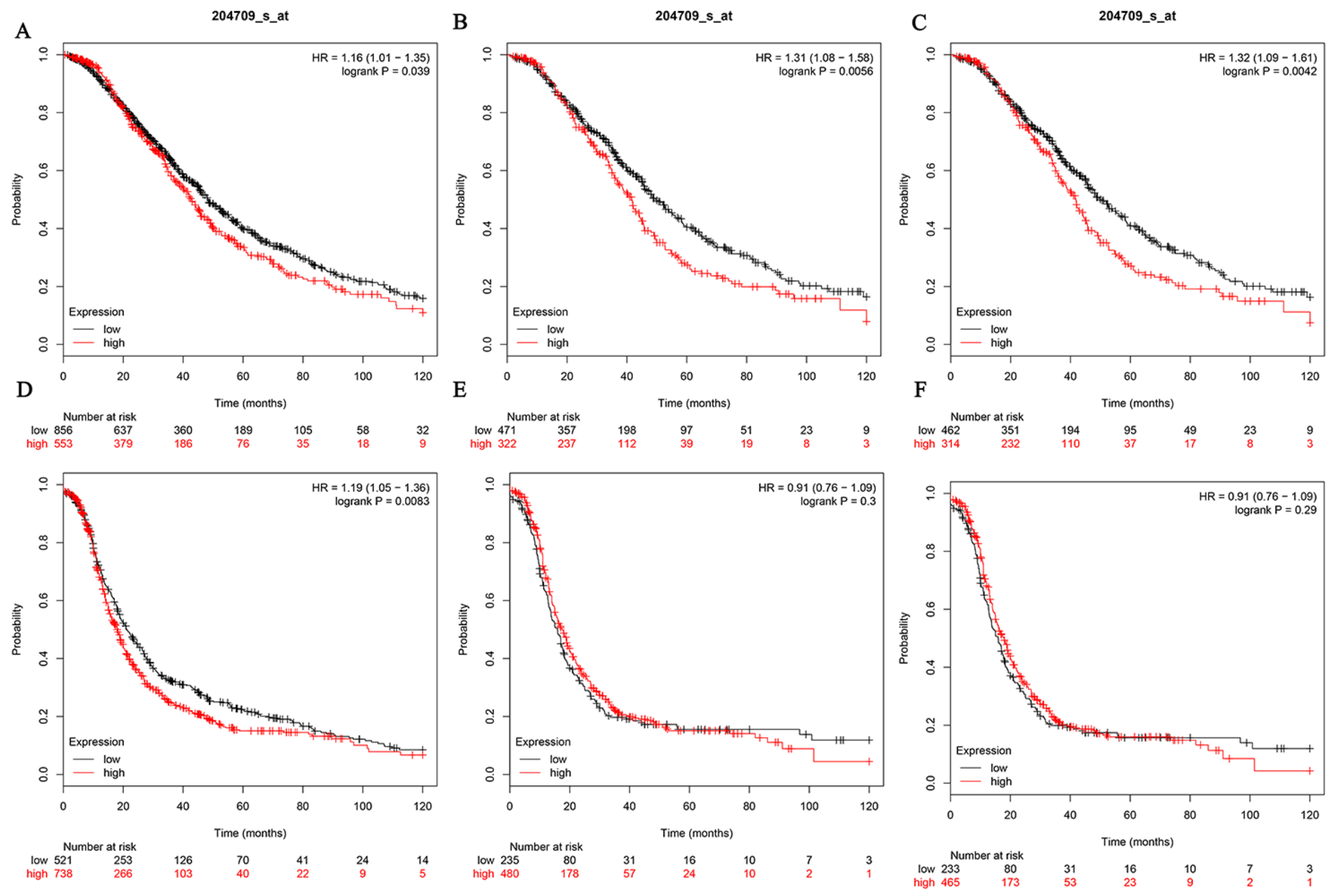

Figure 6

Relationship between KIF23 mRNA expression and chemotherapy in patients with ovarian cancer 

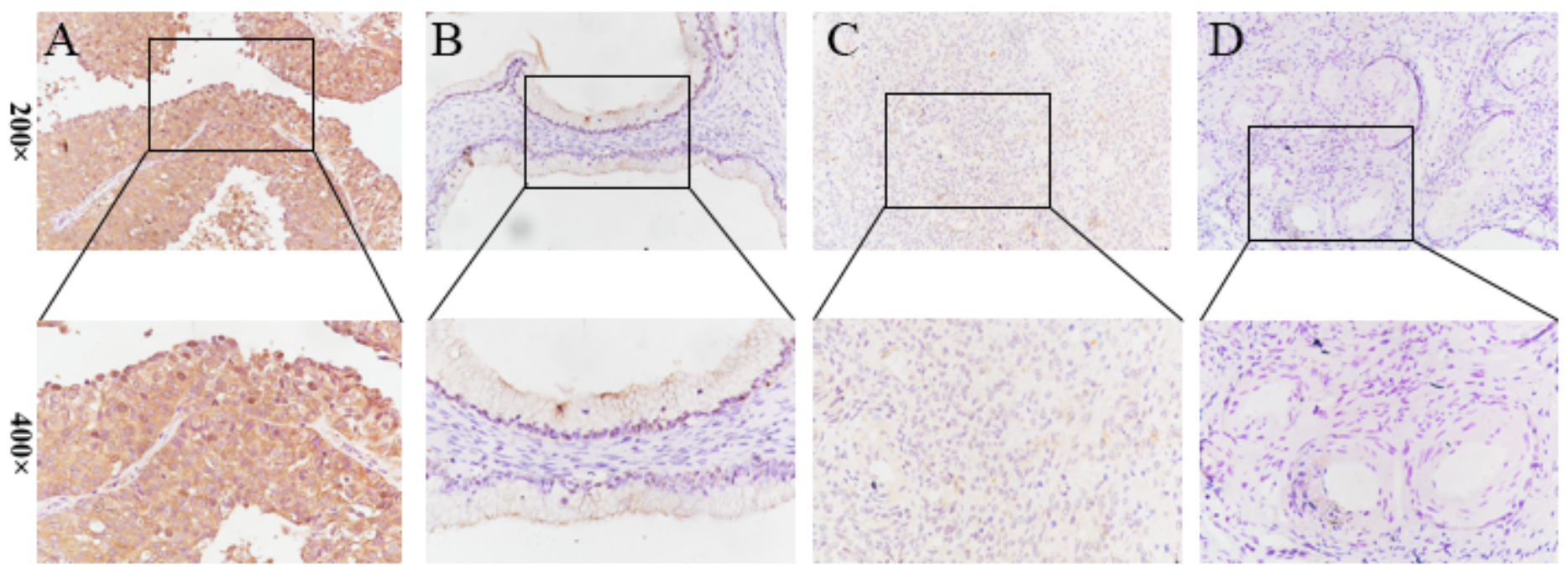

Figure 7

KIF23 immunohistochemistry in ovarian tissues. A. Ovarian epithelial malignant tumor. B. Ovarian epithelial borderline tumor. C. Ovarian epithelial benign tumor. D. Normal ovarian tissues. The lower image is a partial enlargement of the box above. 


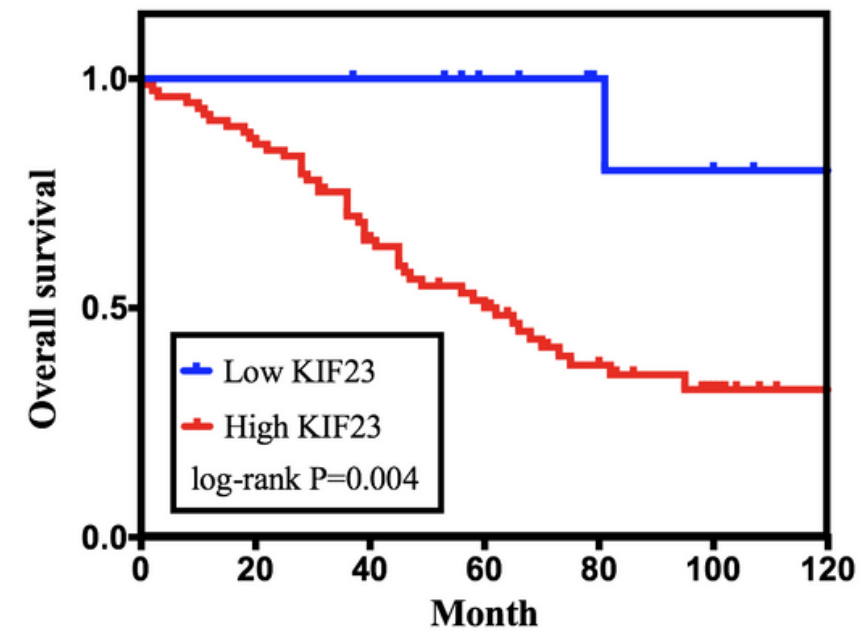

$\mathbf{C}$

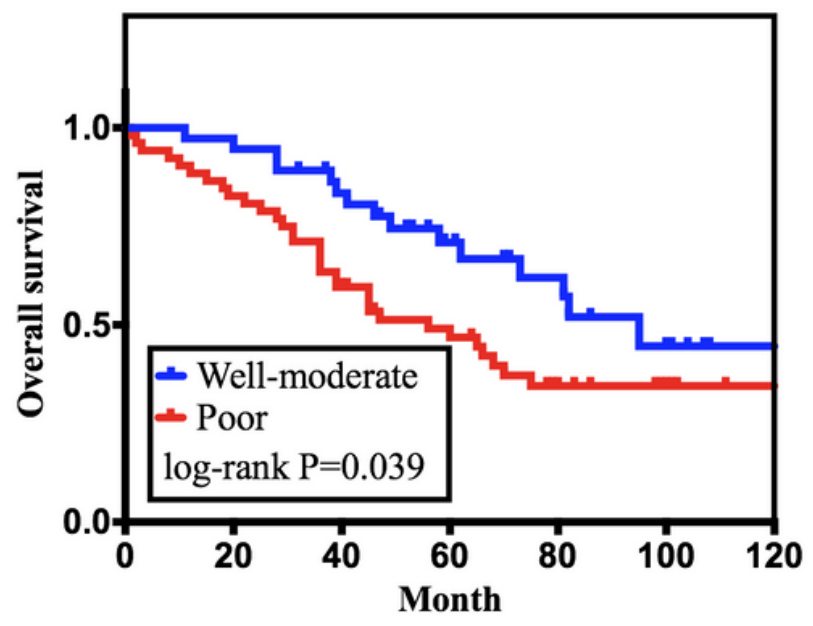

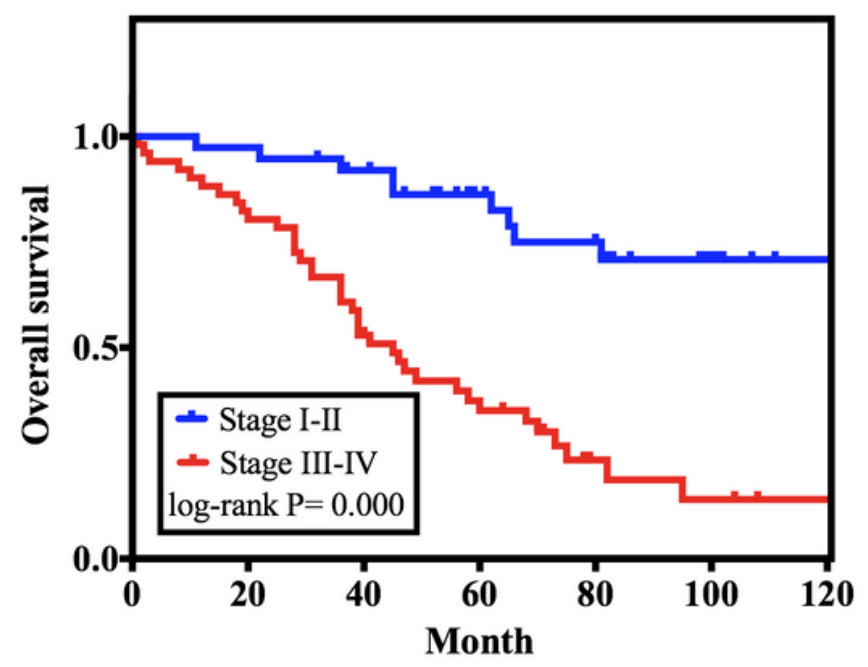

D

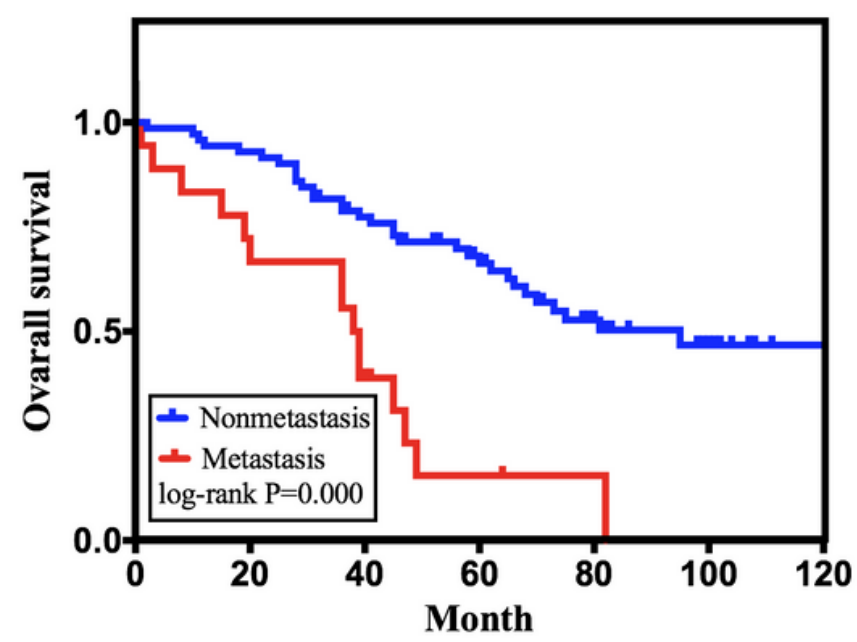

Figure 8

Kaplan-Meier curves for overall survival of patients with epithelial ovarian cancer. A. Correlation of KIF23 expression with overall survival. B. Correlation of clinical stage with overall survival. C. Correlation of the degree of differentiation with overall survival. D. Correlation of lymph node metastasis with overall survival. 
A

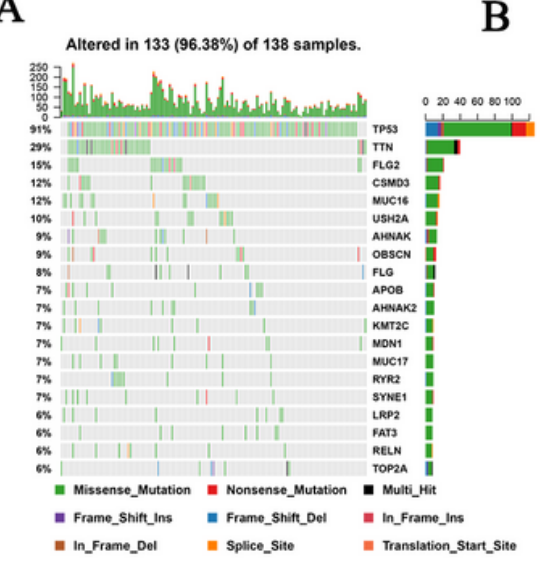

D

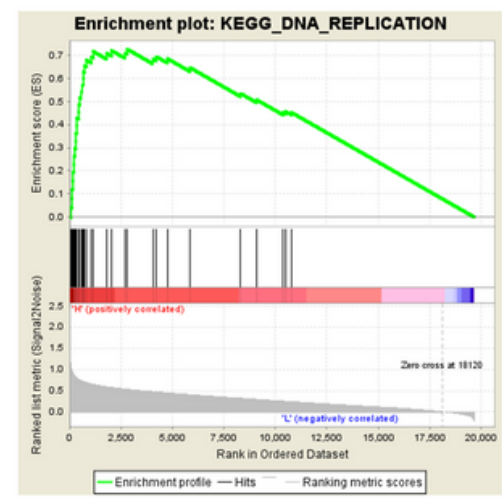

B

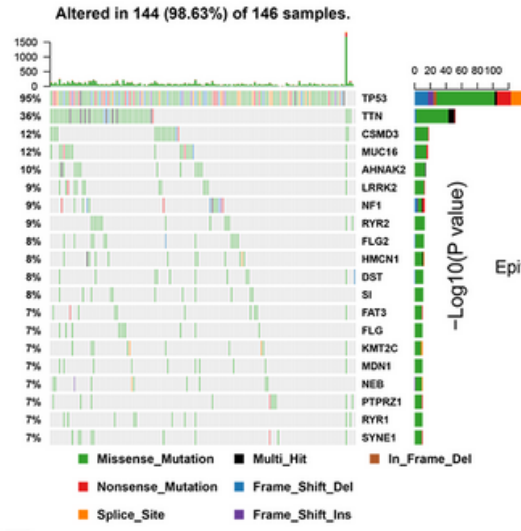

$\mathrm{E}$

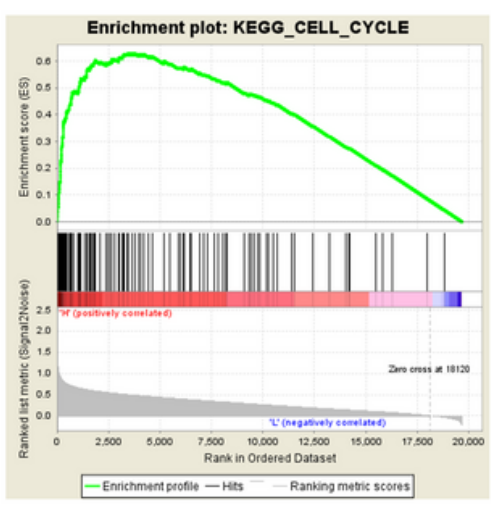

C

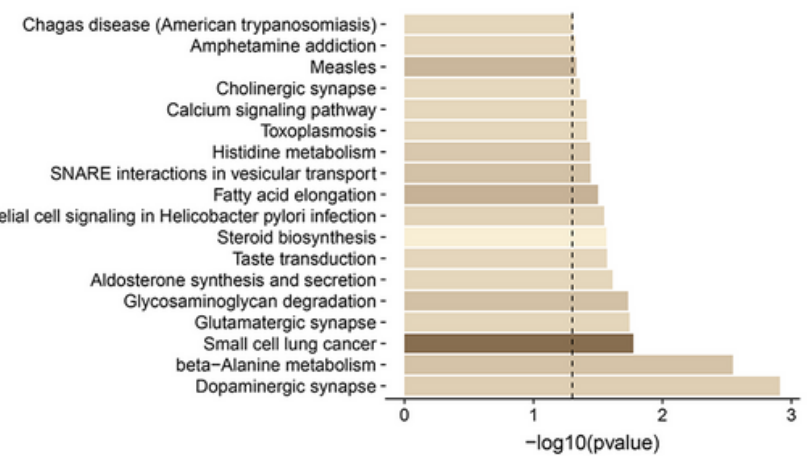

\section{F}

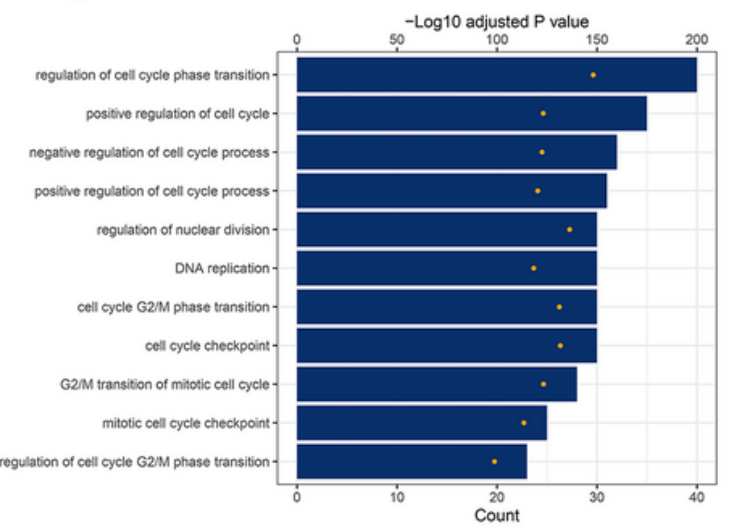

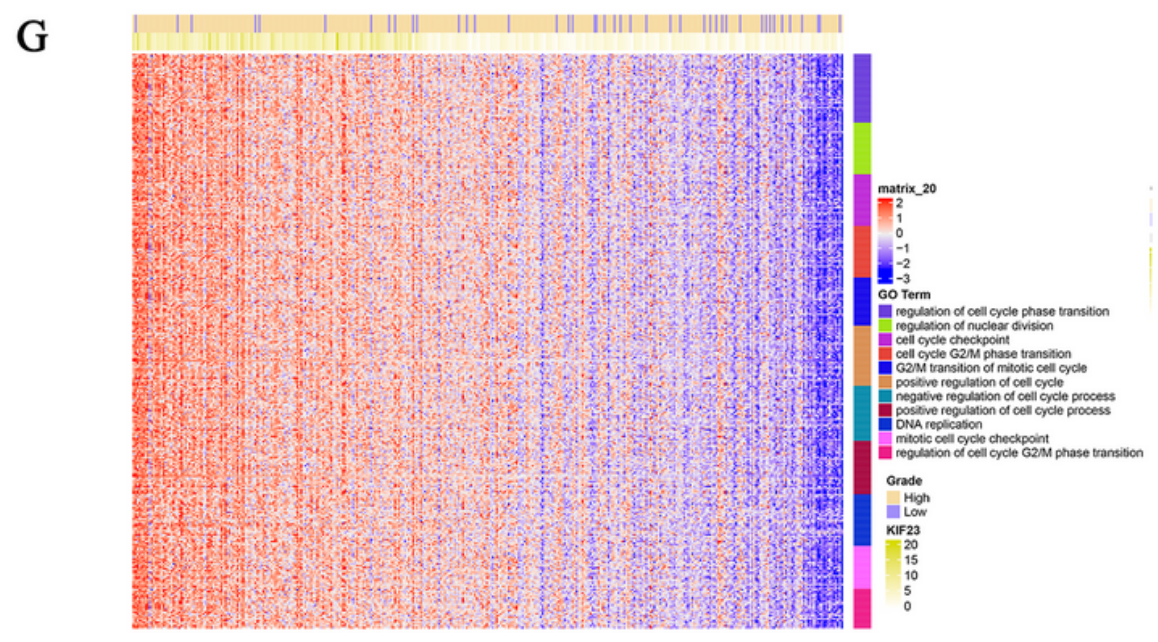

Figure 9

Molecular mechanism of KIF23 in the development of ovarian cancer 